\title{
Error Estimate of a Second Order Accurate Scalar Auxiliary Variable (SAV) Numerical Method for the Epitaxial Thin Film Equation
}

\author{
Qing Cheng ${ }^{1, *}$ and Cheng Wang ${ }^{2}$ \\ ${ }^{1}$ Department of Mathematics, Purdue University, West Lafayette, IN 47907, USA \\ 2 Department of Mathematics, University of Massachusetts, North Dartmouth, \\ MA 02747, USA
}

Received 19 September 2020; Accepted (in revised version) 14 November 2020

\begin{abstract}
A second order accurate (in time) numerical scheme is analyzed for the slope-selection (SS) equation of the epitaxial thin film growth model, with Fourier pseudo-spectral discretization in space. To make the numerical scheme linear while preserving the nonlinear energy stability, we make use of the scalar auxiliary variable (SAV) approach, in which a modified Crank-Nicolson is applied for the surface diffusion part. The energy stability could be derived a modified form, in comparison with the standard Crank-Nicolson approximation to the surface diffusion term. Such an energy stability leads to an $H^{2}$ bound for the numerical solution. In addition, this $H^{2}$ bound is not sufficient for the optimal rate convergence analysis, and we establish a uniform-in-time $H^{3}$ bound for the numerical solution, based on the higher order Sobolev norm estimate, combined with repeated applications of discrete Hölder inequality and nonlinear embeddings in the Fourier pseudo-spectral space. This discrete $H^{3}$ bound for the numerical solution enables us to derive the optimal rate error estimate for this alternate SAV method. A few numerical experiments are also presented, which confirm the efficiency and accuracy of the proposed scheme.
\end{abstract}

AMS subject classifications: 35K30, 35K55, 65K10, 65M12, 65M70

Key words: Epitaxial thin film equation, Fourier pseudo-spectral approximation, the scalar auxiliary variable (SAV) method, Crank-Nicolson temporal discretization, energy stability, optimal rate convergence analysis.

\section{Introduction}

In this article we consider a slope-selection (SS) epitaxial thin film growth equation, which corresponds to the $L^{2}$ gradient flow associated with the following energy func-

*Corresponding author.

Emails: cheng573@puedue.edu (Q. Cheng), cwang1@umassd.edu (C. Wang) 
tional

$$
E(\phi)=\int_{\Omega}\left(\frac{1}{4}\left(|\nabla \phi|^{2}-1\right)^{2}+\frac{\varepsilon^{2}}{2}|\Delta \phi|^{2}\right) d \mathbf{x},
$$

where $\Omega=\left(0, L_{x}\right) \times\left(0, L_{y}\right), u: \Omega \rightarrow \mathbb{R}$ is a periodic height function, and $\varepsilon$ is a constant parameter of transition layer width. In more details, the first nonlinear term represents the Ehrlich-Schwoebel (ES) effect $[23,38-40,54]$, which results in an uphill atom current in the dynamics and the steepening of mounds in the film. The second higher order quadratic term represents the isotropic surface diffusion effect $[39,49]$. In turn, the chemical potential becomes the following variational derivative of the energy

$$
\mu:=\delta_{\phi} E=-\nabla \cdot\left(|\nabla \phi|^{2} \nabla \phi\right)-\Delta \phi+\varepsilon^{2} \Delta^{2} \phi,
$$

and the PDE stands for the $L^{2}$ gradient flow

$$
\partial_{t} \phi=\nabla \cdot\left(|\nabla \phi|^{2} \nabla \phi\right)-\Delta \phi-\varepsilon^{2} \Delta^{2} \phi .
$$

Meanwhile, another epitaxial thin film model has also been extensively studied, with the following energy functional

$$
E(\phi):=\int_{\Omega}\left(-\frac{1}{2} \ln \left(1+|\nabla \phi|^{2}\right)+\frac{\varepsilon^{2}}{2}|\Delta \phi|^{2}\right) d \mathbf{x}
$$

and the dynamical equation is formulated as

$$
\partial_{t} \phi=-\nabla \cdot\left(\frac{\nabla \phi}{1+|\nabla \phi|^{2}}\right)-\varepsilon^{2} \Delta^{2} \phi
$$

This model is referred to as the no-slope-selection (NSS) equation. In fact, the slopeselection energy (1.1) could be viewed an a polynomial approximation to the no-slopeselection energy (1.4), under a small-slope assumption that $|\nabla \phi|^{2} \ll 1$; see the related discussions in $[36,37,39,49]$. A solution to (1.3) exhibits pyramidal structures, where the faces of the pyramids have slopes $|\nabla u| \approx 1$; meanwhile, the no-slope-selection equation (1.5) exhibits mound-like structures, and the slopes of which (on an infinite domain) may grow unbounded $[39,59]$. On the other hand, both solutions have up-down symmetry in the sense that there is no way to distinguish a hill from a valley. This can be altered by adding adsorption/desorption or other dynamics.

The numerical schemes with energy stability have been of great interests, due to the long time nature of the gradient flow coarsening process. There have been many efforts to devise and analyze energy stable numerical schemes for both the SS and NSS equations; see the related references $[1,11,26,35,46,50-53,55,59,61,64]$, etc. In particular, the linear schemes have attracted a great amount of attentions among the energy stable numerical approaches, due to its simplicity of implementation. For the NSS equation (1.5), there have been extensive works of linear, energy stable numerical schemes $[5,7,8,10,13,33$, $35,44,48]$, with up to the third order accuracy in time. Such a nonlinear energy stability 
analysis is based on the following subtle fact: in spite of its complicated form in the denominator, the nonlinear term in the NSS equation (1.5) has automatically bounded higher order derivatives in the $L^{\infty}$ norm.

However, this approach is not applicable to the nonlinear analysis for the SS equation (1.3), due to the polynomial pattern of the nonlinear terms, which in turn requires an $W^{1, \infty}$ bound for the numerical solution at both time steps, $t^{n}$ and $t^{n+1}$, respectively; see the pioneering work [63] of the linear numerical scheme for the SS equation, in which an artificial regularization term, in the form of $A \Delta\left(\phi^{n+1}-\phi^{n}\right)$, has to be included for the sake of energy stability. A theoretical analysis has been provided in [43] for the first order accurate (in time) scheme, to justify the lower bound of the artificial parameter $A$. Various estimates have indicated that, the theoretical value of $A$ is of order $\mathcal{O}\left(\varepsilon^{-2} \ln \varepsilon\right)$ for the first order scheme, while such an artificial regularization parameter has to be of order $\mathcal{O}\left(\varepsilon^{-m_{0}}\right)$, with $m_{0} \geq 10$, for the temporally second order schemes [41,42].

It is clear that, such a singular dependence of the theoretical value of artificial regularization parameter on $\varepsilon$ comes from the $W^{1, \infty}$ estimate for the numerical solution. To avoid a singular artificial regularization parameter in a linear numerical scheme, while preserving an energy stability, the scalar auxiliary variable (SAV) approach for various gradient flows has attracted many attentions in recent years $[56-58,67]$. To overcome the difficulty associated with the nonlinearity, the energy functional is split into two parts: a nonlinear energy functional with a uniform lower bound, combined with a quadratic surface diffusion energy with constant-coefficients. In turn, the elevated nonlinear energy part (which contains a global constant to make its value positive) is rewritten as a quadratic term, not in terms of the original physical variable, but in terms of an artificially-introduced auxiliary variable. As a result, linear schemes could be derived for the gradient flow reformulated in the quadratic nonlinear energy and the surface diffusion energy, so that both the unique solvability and modified energy stability could be theoretically justified for the linear schemes. Also notice that, such an energy estimate is in terms of the reformulated energy functional, not in terms of the original energy functional.

An application of the SAV method to the SS equation (1.3) has been reported in a recent article [17], combined with the Fourier pseudo-spectral spatial approximation. The unique solvability, numerical implementation process and a modified energy stability have been presented for the second order accurate (in time) SAV scheme, with CrankNicolson temporal discretization. However, an optimal rate convergence analysis seems challenging for the proposed scheme in [17]. In particular, a discrete $W^{1, \infty}$ bound for the numerical solution is necessary to pass through the error estimate. On thr other hand, the discrete energy stability (established in [17]) leads to a uniform-in-time discrete $H^{2}$ bound for the numerical solution, while this bound is not sufficient to ensure a $W^{1, \infty}$ bound for the numerical solution.

In this article, we present an optimal rate convergence analysis and error estimate for the second order SAV scheme to the SS equation (1.3), with a slight modification of the surface diffusion coefficients. In more details, the standard Crank-Nicolson approximation to the surface diffusion term is replaced by an alternate one, with $3 / 4$ and $1 / 4$ 
coefficient distribution at time steps $t^{n+1}, t^{n-1}$, respectively. Such an alternate CrankNicolson approximation has been reported in [16,19,20,30-32] for the Cahn-Hilliard and other related gradient model, and great success and advantages over the standard CrankNicolson have been observed. With an application of this approximation to the second order SAV scheme for the SS equation (1.3), a modified energy stability could also be proved in a careful way, so that a uniform $\mathrm{H}^{2}$ bound for the numerical solution (of the phase variable) is available. In addition, a higher order $H^{3}$ estimate could also be derived, with the help of various discrete Sobolev inequality in the Fourier pseudo-spectral space. With such an $\mathrm{H}^{3}$ bound at hand, we are able to control the nonlinear chemical potential error function, in the Fourier pseudo-spectral space. In addition, one nonlinear error inner product could be cancelled between the error evolutionary equations for the original phase variable and the one for the introduced auxiliary variable. These preliminary estimates enable one to obtain an optimal rate $\left(\mathcal{O}\left(\Delta t^{2}+h^{m}\right)\right)$ convergence analysis for the proposed numerical scheme in the energy norm, i.e., in the $\ell^{\infty}\left(0, T ; H_{N}^{2}\right) \cap \ell^{2}\left(0, T ; H_{N}^{4}\right)$ norm. In particular, the aliasing error control techniques have to be applied in the nonlinear error estimate associated with the 4-Laplacian term.

The outline of the paper is given as follows. In Section 2 we present the numerical scheme. First we review the Fourier pseudo-spectral approximation in space and recall an aliasing error control technique. Then we recall the SAV numerical scheme, and prove its energy stability in a modified way. In addition, a uniform-in-time $H^{3}$ bound for the numerical solution is established in Section 3, and an optimal rate convergence analysis is provided in Section 4. Some numerical results are presented in Section 5. Finally, some concluding remarks are made in Section 6.

\section{The numerical scheme}

\subsection{Review of Fourier pseudo-spectral approximations}

The Fourier pseudo-spectral method is also referred as the Fourier collocation spectral method. It is closely related to the Fourier spectral method, but complements the basis by an additional pseudo-spectral basis, which allows to represent functions on a quadrature grid. This simplifies the evaluation of certain operators, and can considerably speed up the calculation when using fast algorithms such as the fast Fourier transform (FFT); see the related descriptions in $[2,12,14,16,28,29,34,65,66]$, etc.

To simplify the notation in our pseudo-spectral analysis, we assume that the domain is given by $\Omega=(0,1)^{2}, N_{x}=N_{y}=: N \in \mathbb{N}$ and $N \cdot h=1$. We further assume that $N$ is odd:

$$
N=2 K+1 \text { for some } K \in \mathbb{N} \text {. }
$$

The analyses for more general cases are a bit more tedious, but can be carried out without essential difficulty. The spatial variables are evaluated on the standard $2 \mathrm{D}$ numerical grid $\Omega_{N}$, which is defined by grid points $\left(x_{i}, y_{j}\right)$, with $x_{i}=i h, y_{j}=j h, 0 \leq i, j \leq 2 K+1$. 
We define the grid function space

$$
\mathcal{G}_{N}:=\left\{f: \mathbb{Z}^{2} \rightarrow \mathbb{R} \mid f \text { is } \Omega_{N} \text {-periodic }\right\} .
$$

Given any periodic grid functions $f, g \in \mathcal{G}_{N}$, the $\ell^{2}$ inner product and norm are defined as

$$
\langle f, g\rangle:=h^{2} \sum_{i, j, k=0}^{N-1} f_{i, j} \cdot g_{i, j}, \quad\|f\|_{2}:=\sqrt{\langle f, f\rangle} .
$$

The zero-mean grid function subspace is denoted as

$$
\stackrel{\mathcal{G}}{N}_{N}:=\left\{f \in \mathcal{G}_{N} \mid\langle f, 1\rangle=: \bar{f}=0\right\} .
$$

For $f \in \mathcal{G}_{N}$, we have the discrete Fourier expansion

$$
f_{i, j}=\sum_{\ell, m=-K}^{K} \hat{f}_{\ell, m}^{N} \exp \left(2 \pi \mathrm{i}\left(\ell x_{i}+m y_{j}\right)\right),
$$

where the discrete Fourier coefficients are given by

$$
\hat{f}_{\ell, m}^{N}:=h^{2} \sum_{i, j,=0}^{N-1} f_{i, j, k} \exp \left(-2 \pi \mathrm{i}\left(\ell x_{i}+m x_{j}\right)\right) .
$$

The collocation Fourier spectral first and second order derivatives of $f$ are defined as

$$
\begin{aligned}
& \mathcal{D}_{x} f_{i, j}:=\sum_{\ell, m=-K}^{K}(2 \pi \mathrm{i} \ell) \hat{f}_{\ell, m}^{N} \exp \left(2 \pi \mathrm{i}\left(\ell x_{i}+m y_{j}\right)\right), \\
& \mathcal{D}_{x}^{2} f_{i, j}:=\sum_{\ell, m=-K}^{K}\left(-4 \pi^{2} \ell^{2}\right) \hat{f}_{\ell, m}^{N} \exp \left(2 \pi \mathrm{i}\left(\ell x_{i}+m y_{j}\right)\right) .
\end{aligned}
$$

The differentiation operators in the $y$ direction, $\mathcal{D}_{y}$ and $\mathcal{D}_{y}^{2}$, can be defined in the same fashion. In turn, the discrete Laplacian, gradient and divergence operators are given by

$$
\Delta_{N} f:=\left(\mathcal{D}_{x}^{2}+\mathcal{D}_{y}^{2}\right) f, \quad \nabla_{N} f:=\left(\begin{array}{c}
\mathcal{D}_{x} f \\
\mathcal{D}_{y} f
\end{array}\right), \quad \nabla_{N} \cdot\left(\begin{array}{c}
f_{1} \\
f_{2}
\end{array}\right):=\mathcal{D}_{x} f_{1}+\mathcal{D}_{y} f_{2},
$$

at the point-wise level. It is straightforward to verify that

$$
\nabla_{N} \cdot \nabla_{N} f=\Delta_{N} f
$$

See the derivations in the related references $[2,3,27]$. 
Definition 2.1. Suppose that the grid function $f \in \mathcal{G}_{N}$ has the discrete Fourier expansion (2.3). Its spectral extension into the trigonometric polynomial space $\mathcal{P}_{K}$ (the space of trigonometric polynomials of degree at most $K$ ) is defined as

$$
f_{S}(x, y)=\sum_{\ell, m=-K}^{K} \hat{f}_{\ell, m}^{N} \exp (2 \pi \mathrm{i}(\ell x+m y)) .
$$

We write $S_{N}(f)=f_{S}$ and call $S_{N}: \mathcal{G}_{N} \rightarrow \mathcal{P}_{K}$ the spectral interpolation operator. Suppose $g \in$ $C_{\text {per }}(\Omega, \mathbb{R})$. We define the grid projection $Q_{N}: C_{\text {per }}(\Omega, \mathbb{R}) \rightarrow \mathcal{G}_{N}$ via

$$
Q_{N}(g)_{i, j}:=g\left(x_{i}, y_{j}\right) \text {. }
$$

The resultant grid function may, of course, be expressed as a discrete Fourier expansion:

$$
Q_{N}(g)_{i, j}=\sum_{\ell, m=-K}^{K}{\widehat{Q_{N}(g)}}_{\ell, m}^{N} \exp \left(2 \pi \mathrm{i}\left(\ell x_{i}+m y_{j}\right)\right)
$$

We define the de-aliasing operator $R_{N}: C_{\text {per }}(\Omega, \mathbb{R}) \rightarrow \mathcal{P}_{K}$ via $R_{N}:=S_{N}\left(Q_{N}\right)$. In other words,

$$
R_{N}(g)(x, y)=\sum_{\ell, m=-K}^{K}{\widehat{Q_{N}(g)}}_{\ell, m}^{N} \exp (2 \pi \mathrm{i}(\ell x+m y))
$$

Finally, for any $g \in L^{2}(\Omega, \mathbb{R})$, we define the (standard) Fourier projection operator $P_{N}: L^{2}(\Omega, \mathbb{R}) \rightarrow$ $\mathcal{P}_{K}$ via

$$
P_{N}(g)(x, y)=\sum_{\ell, m=-K}^{K} \hat{g}_{\ell, m} \exp (2 \pi \mathrm{i}(\ell x+m y))
$$

where

$$
\hat{g}_{\ell, m}=\int_{\Omega} g(x, y) \exp (-2 \pi \mathrm{i}(\ell x+m y)) d \mathbf{x},
$$

are the (standard) Fourier coefficients.

To overcome a key difficulty associated with the $H^{m}$ bound of the nonlinear term obtained by collocation interpolation, the following lemma is introduced. The case of $r=0$ was proven in an earlier work [21,22], and the case of $r \geq 1$ was analyzed in a recent article [29].

Lemma 2.1. Suppose that $m$ and $K$ are non-negative integers, and, as before, assume that $N=$ $2 K+1$. For any $\varphi \in \mathcal{P}_{m K}$ in $\mathbb{R}^{d}$, we have the estimate

$$
\left\|R_{N}(\varphi)\right\|_{H^{r}} \leq m^{\frac{d}{2}}\|\varphi\|_{H^{r}}
$$

for any non-negative integer $r$. 
Furthermore, to facilitate the analysis in later sections, we introduce an operator $L_{N}$ as $L_{N} f:=\varepsilon^{2} \Delta_{N}^{2} f$, for any $f \in \mathcal{G}_{N}$. In addition, a fractional operator is similarly introduced as $L_{N}^{\frac{1}{2}} f:=\varepsilon\left(-\Delta_{N}\right) f$, for any $f \in \mathcal{G}_{N}$.

The following summation-by-parts formulas are valid (see the related discussions in $[5,10,28,29])$ : for any periodic grid functions $f, g \in \mathcal{G}_{N}$,

$$
\left\langle f, \Delta_{N} g\right\rangle=-\left\langle\nabla_{N} f, \nabla_{N} g\right\rangle, \quad\left\langle f, \Delta_{N}^{2} g\right\rangle=\left\langle\Delta_{N} f, \Delta_{N} g\right\rangle .
$$

Similarly, the following identity could be derived in the same manner:

$$
\left\langle f, L_{N} g\right\rangle=\left\langle L_{N}^{\frac{1}{2}} f, L_{N}^{\frac{1}{2}} g\right\rangle, \quad \forall f, g \in \mathcal{G}_{N} .
$$

In addition to the standard $\ell^{2}$ norm, we also introduce the $\ell^{p}, 1 \leq p<\infty$, and $\ell^{\infty}$ norms for a grid function $f \in \mathcal{G}_{N}$ :

$$
\|f\|_{\infty}:=\max _{i, j, k}\left|f_{i, j}\right|, \quad\|f\|_{p}:=\left(h^{2} \sum_{i, j=0}^{N-1}\left|f_{i, j}\right|^{p}\right)^{\frac{1}{p}}, \quad 1 \leq p<\infty
$$

The discrete $H^{1}$ and $H^{2}$ norms are introduced as

$$
\|f\|_{H_{N}^{1}}^{2}=\|f\|_{2}^{2}+\left\|\nabla_{N} f\right\|_{2}^{2}, \quad\|f\|_{H_{N}^{2}}^{2}=\|f\|_{H_{N}^{1}}^{2}+\left\|\Delta_{N} f\right\|_{2}^{2} .
$$

For any periodic grid function $\phi \in \mathcal{G}_{N}$, the discrete energy is defined as

$$
E_{N}(\phi):=\frac{1}{4}\left\|\nabla_{N} \phi\right\|_{4}^{4}-\frac{1}{2}\left\|\nabla_{N} \phi\right\|_{2}^{2}+\frac{\varepsilon^{2}}{2}\left\|\Delta_{N} \phi\right\|_{2}^{2} .
$$

The following result corresponds to a discrete Sobolev embedding from $H_{N}^{2}$ to $W_{N}^{1,6}$ in the pseudo-spectral space. Similar discrete embedding estimates, in the lower order ones, could be found in Lemma 2.1 of [16]; also see the related results $[24,25]$ in the finite difference version. A direct calculation is not able to derive these inequalities; instead, a discrete Fourier analysis has to be applied in the derivation; the details of the proof has been provided in a recent work [15].

Proposition 2.1 ([15]). For any periodic grid function $f$, we have

$$
\left\|\nabla_{N} f\right\|_{6} \leq C\left\|\Delta_{N} f\right\|_{2}
$$

for some constant $C$ only dependent on $\Omega$.

\subsection{The fully discrete numerical scheme}

The energy (1.1) is decomposed into two parts:

$$
E(\phi)=E_{1}(\phi)+\frac{1}{2}(\phi, L \phi), \quad E_{1}(\phi)=\int_{\Omega}\left\{\frac{1}{4}|\nabla \phi|^{4}-\frac{1}{2}|\nabla \phi|^{2}+\frac{1}{4}\right\} d \mathbf{x}, \quad L \phi=\varepsilon^{2} \Delta^{2} \phi .
$$


In particular, due to the point-wise quadratic inequality

$$
\frac{1}{4}|\nabla \phi|^{4}-\frac{1}{2}|\nabla \phi|^{2}+\frac{1}{4} \geq 0,
$$

we conclude that $E_{1}(\phi)$ have a well-established lower bound:

$$
E_{1}(\phi) \geq|\Omega| \text {. }
$$

In turn, the nonlinear chemical potential becomes

$$
N(\phi):=\delta_{\phi} E_{1}=-\nabla \cdot\left(|\nabla \phi|^{2} \nabla \phi\right)+\Delta \phi .
$$

Therefore, with an introduction of a scalar auxiliary variable

$$
r:=\sqrt{E_{1}(\phi)},
$$

the original SS equation (1.3) could be rewritten as the following system:

$$
\left\{\begin{array}{l}
\phi_{t}=\Delta\left(\frac{r}{\sqrt{E_{1}(\phi)}} N(\phi)+L \phi\right), \\
r_{t}=\frac{1}{2 \sqrt{E_{1}(\phi)}} \int_{\Omega} N(\phi) \phi_{t} d \mathbf{x} .
\end{array}\right.
$$

Based on this reformulation, the fully discrete second order SAV scheme is proposed as follows, with Fourier pseudo-spectral spatial approximation:

$$
\left\{\begin{array}{l}
\frac{\phi^{n+1}-\phi^{n}}{\Delta t}=-\frac{r^{n+1 / 2}}{\sqrt{E_{1, N}\left(\hat{\phi}^{n+1 / 2}\right)}} N_{N}\left(\hat{\phi}^{n+1 / 2}\right)-L_{N}\left(\frac{3}{4} \phi^{n+1}+\frac{1}{4} \phi^{n-1}\right), \\
\frac{r^{n+1}-r^{n}}{\Delta t}=\frac{1}{2 \sqrt{E_{1, N}\left(\hat{\phi}^{n+1 / 2}\right)}}\left\langle N_{N}\left(\hat{\phi}^{n+1 / 2}\right), \frac{\phi^{n+1}-\phi^{n}}{\Delta t}\right\rangle,
\end{array}\right.
$$

in which

$$
N_{N}(\phi):=-\nabla_{N} \cdot\left(\left|\nabla_{N} \phi\right|^{2} \nabla_{N} \phi\right)+\Delta_{N} \phi, \quad L_{N} \phi=\varepsilon^{2} \Delta_{N}^{2} \phi, \quad r^{n+1 / 2}=\frac{1}{2}\left(r^{n+1}+r^{n}\right),
$$

and a second order explicit extrapolation is applied to obtain

$$
\hat{\phi}^{n+1 / 2}=\frac{3}{2} \phi^{n}-\frac{1}{2} \phi^{n-1} .
$$

The discrete nonlinear energy functional is introduced as

$$
E_{1, N}(\phi):=\frac{1}{4}\left\|\nabla_{N} \phi\right\|_{4}^{4}-\frac{1}{2}\left\|\nabla_{N} \phi\right\|_{2}^{2}+\frac{1}{4}|\Omega|,
$$


similar to the notation in (2.16).

Since (2.24) is a two-step numerical method, a "ghost" point extrapolation for $\phi^{-1}$ is useful. To preserve the second order accuracy in time, we apply the following approximation:

$$
\phi^{-1}=\phi^{0}+\Delta t \mu^{0}, \quad \mu^{0}:=-\nabla_{N} \cdot\left(\left|\nabla_{N} \phi^{0}\right|^{2} \nabla_{N} \phi^{0}\right)+\Delta_{N} \phi^{0}+\varepsilon^{2} \Delta_{N}^{2} \phi^{0} .
$$

A careful Taylor expansion indicates an $\mathcal{O}\left(\Delta t^{2}+h^{m}\right)$ accuracy for such an approximation:

$$
\left\|\phi^{-1}-\Phi^{-1}\right\|_{2} \leq C\left(\Delta t^{2}+h^{m}\right) .
$$

In turn, we take

$$
r^{0}:=\sqrt{E_{1, N}\left(\phi^{0}\right)}, \quad r^{-1}:=\sqrt{E_{1, N}\left(\phi^{-1}\right)}
$$

\subsection{Unique solvability and efficient numerical solver for the numerical scheme}

In this section we analyze the unique solvability of the proposed SAV scheme (2.24). From (2.24a), one can get

$$
\left(I+\frac{3}{4} \Delta t L_{N}\right) \phi^{n+1}=-\Delta t \frac{r^{n+1 / 2}}{\sqrt{E_{1, N}\left(\hat{\phi}^{n+1 / 2}\right)}} N_{N}\left(\hat{\phi}^{n+1 / 2}\right)-\frac{1}{4} \Delta t L_{N} \phi^{n-1}+\phi^{n} .
$$

Define

$$
A_{N}=I+\frac{3}{4} \Delta t L_{N}
$$

so that the following identity is valid:

$$
\phi^{n+1}=-\Delta t \frac{r^{n+1 / 2}}{\sqrt{E_{1, N}\left(\hat{\phi}^{n+1 / 2}\right)}} A_{N}^{-1} N_{N}\left(\hat{\phi}^{n+1 / 2}\right)+A_{N}^{-1}\left(\phi^{n}-\frac{1}{4} \Delta t L_{N} \phi^{n-1}\right) .
$$

From (2.24b), we see that

$$
r^{n+1}=r^{n}+\frac{1}{2 \sqrt{E_{1, N}\left(\hat{\phi}^{n+1 / 2}\right)}}\left\langle N_{N}\left(\hat{\phi}^{n+1 / 2}\right), \phi^{n+1}-\phi^{n}\right\rangle .
$$

A substitution of (2.28) into (2.27) gives

$$
\begin{aligned}
& \left(I+\frac{3}{4} \Delta t L_{N}\right) \phi^{n+1}+\frac{N_{N}\left(\hat{\phi}^{n+1 / 2}\right)}{4 E_{1, N}\left(\hat{\phi}^{n+1 / 2}\right)} \Delta t\left\langle N_{N}\left(\hat{\phi}^{n+1 / 2}\right), \phi^{n+1}\right\rangle \\
= & -\frac{\Delta t N_{N}\left(\hat{\phi}^{n+1 / 2}\right)}{\sqrt{E_{1, N}\left(\hat{\phi}^{n+1 / 2}\right)}}\left(r^{n}-\frac{1}{4 \sqrt{E_{1, N}\left(\hat{\phi}^{n+1 / 2}\right)}}\left\langle N_{N}\left(\hat{\phi}^{n+1 / 2}\right), \phi^{n}\right\rangle\right)+\phi^{n}-\frac{1}{4} \Delta t L_{N} \phi^{n-1} .
\end{aligned}
$$


Let $g_{N}^{n}$ denotes the right-hand of the above equation, then it becomes

$$
A_{N} \phi^{n+1}+\frac{N_{N}\left(\hat{\phi}^{n+1 / 2}\right)}{4 E_{1, N}\left(\hat{\phi}^{n+1 / 2}\right)} \Delta t\left\langle N_{N}\left(\hat{\phi}^{n+1 / 2}\right), \phi^{n+1}\right\rangle=g_{N}^{n} .
$$

Multiplying both sides by $A_{N}^{-1}$ implies that

$$
\phi^{n+1}+\frac{1}{4 E_{1, N}\left(\hat{\phi}^{n+1 / 2}\right)} \Delta t\left\langle N_{N}\left(\hat{\phi}^{n+1 / 2}\right), \phi^{n+1}\right\rangle \cdot A_{N}^{-1} N_{N}\left(\hat{\phi}^{n+1 / 2}\right)=A_{N}^{-1} g_{N}^{n} .
$$

Denote $L H S=\left\langle N_{N}\left(\hat{\phi}^{n+1 / 2}\right), \phi^{n+1}\right\rangle$, a scalar value. Taking a discrete inner product with (2.29) by $N_{N}\left(\hat{\phi}^{n+1 / 2}\right)$ leads to

$$
\begin{aligned}
& \left\langle N_{N}\left(\hat{\phi}^{n+1 / 2}\right), \phi^{n+1}\right\rangle+\frac{\Delta t}{4 E_{1, N}\left(\hat{\phi}^{n+1 / 2}\right)} \cdot L H S \cdot\left\langle N_{N}\left(\hat{\phi}^{n+1 / 2}\right), A_{N}^{-1} N_{N}\left(\hat{\phi}^{n+1 / 2}\right)\right\rangle \\
= & \left\langle N_{N}\left(\hat{\phi}^{n+1 / 2}\right), A_{N}^{-1} g_{N}^{n}\right\rangle .
\end{aligned}
$$

Then we arrive at

$$
\begin{aligned}
& \left(1+\frac{\Delta t}{4 E_{1, N}\left(\hat{\phi}^{n+1 / 2}\right)} \cdot\left\langle N_{N}\left(\hat{\phi}^{n+1 / 2}\right), A_{N}^{-1} N_{N}\left(\hat{\phi}^{n+1 / 2}\right)\right\rangle\right) \cdot \text { LHS } \\
= & \left\langle N_{N}\left(\hat{\phi}^{n+1 / 2}\right), A_{N}^{-1} g_{N}^{n}\right\rangle .
\end{aligned}
$$

In addition, we notice that

$$
\left\langle N_{N}\left(\hat{\phi}^{n+1 / 2}\right), A_{N}^{-1} N_{N}\left(\hat{\phi}^{n+1 / 2}\right)\right\rangle \geq 0,
$$

since all the eigenvalues of the symmetric operator $A_{N}^{-1}$ are non-negative. As a direct consequence, the coefficient on the left hand side of (2.30) is positive, so that the value of LHS is uniquely solvable. Going back (2.29), the numerical solution $\phi^{n+1}$ is uniquely determined:

$$
\phi^{n+1}=-\frac{\Delta t}{4 E_{1, N}\left(\hat{\phi}^{n+1 / 2}\right)} \cdot L H S \cdot A_{N}^{-1} N_{N}\left(\hat{\phi}^{n+1 / 2}\right)+A_{N}^{-1} g_{N}^{n} .
$$

Furthermore, a substitution of $\phi^{n+1}$ into (2.28) gives the numerical value of $r^{n+1}$.

Theorem 2.1. Given $\phi^{n}, \phi^{n-1} \in \mathcal{G}_{N}$, two scalar values $r^{n}, r^{n-1}$, with $\overline{\phi^{n}}=\overline{\phi^{n-1}}$, there exists a unique solution $\phi^{n+1} \in \mathcal{G}_{N}$ for the numerical schemes (2.24). The scheme is mass conservative, i.e., $\overline{\phi^{k}} \equiv \overline{\phi^{0}}:=\beta_{0}$, for any $k \geq 0$, provided that $\overline{\phi^{-1}}=\overline{\phi^{0}}=\beta_{0}$.

Proof. The unique solvability comes from the derived identities (2.28), (2.30) and (2.32). In addition, the mass conservation property is a direct consequence of a summation of (2.24a) over $\Omega$, which is turn leads to

$$
\overline{\phi^{n+1}}=\overline{\phi^{n}}+\Delta t \Delta_{N}\left(\frac{r^{n+1 / 2}}{\left.\sqrt{E_{1, N}\left(\hat{\phi}^{n+1 / 2}\right)} N_{N}\left(\hat{\phi}^{n+1 / 2}\right)+L_{N}\left(\frac{3}{4} \phi^{n+1}+\frac{1}{4} \phi^{n-1}\right)\right)}=\overline{\phi^{n}},\right.
$$


with the fact that $\overline{\Delta_{N} f}=0, \forall f \in \mathcal{G}_{N}$, has been applied. An application of induction implies that $\overline{\phi^{k}}=\beta_{0}$, for any $k \geq 0$, provided that $\overline{\phi^{-1}}=\overline{\phi^{0}}=\beta_{0}$. This completes the proof of Theorem 2.1.

\subsection{Modified energy stability for the numerical scheme}

Theorem 2.2. For $k \geq 1$, define the discrete modified energy

$$
\mathcal{E}_{N}\left(\phi^{k+1}, \phi^{k}, r^{k+1}\right):=\frac{1}{2}\left\|L_{N}^{\frac{1}{2}} \phi^{k+1}\right\|_{2}^{2}+\left|r^{k+1}\right|^{2}+\frac{1}{8}\left\|L_{N}^{\frac{1}{2}}\left(\phi^{k+1}-\phi^{k}\right)\right\|_{2}^{2} .
$$

Solution of the numerical scheme (2.24) satisfies the following dissipation properties

$$
\mathcal{E}_{N}\left(\phi^{k+1}, \phi^{k}, r^{k+1}\right) \leq \mathcal{E}_{N}\left(\phi^{k}, \phi^{k-1}, r^{k}\right) .
$$

Proof. We begin with a rewritten form of the numerical scheme (2.24):

$$
\left\{\begin{array}{l}
\frac{\phi^{n+1}-\phi^{n}}{\Delta t}=-\mu_{N}^{n+1 / 2}, \\
\mu_{N}^{n+1 / 2}=L_{N}\left(\frac{3}{4} \phi^{n+1}+\frac{1}{4} \phi^{n-1}\right)+\frac{r^{n+1 / 2}}{\sqrt{E_{1, N}\left(\hat{\phi}^{n+1 / 2}\right)}} N_{N}\left(\hat{\phi}^{n+1 / 2}\right) \\
\frac{r^{n+1}-r^{n}}{\Delta t}=\frac{1}{2 \sqrt{E_{1, N}\left(\hat{\phi}^{n+1 / 2}\right)}}\left\langle N_{N}\left(\hat{\phi}^{n+1 / 2}\right), \frac{\phi^{n+1}-\phi^{n}}{\Delta t}\right\rangle .
\end{array}\right.
$$

Subsequently, taking discrete inner product with (2.36a) by $\mu_{N}^{n+1}$, with (2.36b) by $-\left(\phi^{n+1}-\right.$ $\left.\phi^{n}\right)$, with (2.36c) by $2 r^{n+1 / 2}=r^{n+1}+r^{n}$, we have

$$
\begin{aligned}
\left\langle\phi^{n+1}-\phi^{n}, \mu_{N}^{n+1 / 2}\right\rangle=-\Delta t & \left\|\mu_{N}^{n+1 / 2}\right\|_{2}^{2}, \\
-\left\langle\phi^{n+1}-\phi^{n}, \mu_{N}^{n+1 / 2}\right\rangle=- & \left\langle L_{N}\left(\frac{3}{4} \phi^{n+1}+\frac{1}{4} \phi^{n-1}\right), \phi^{n+1}-\phi^{n}\right\rangle \\
& +\frac{r^{n+1 / 2}}{\sqrt{E_{1, N}\left(\hat{\phi}^{n+1 / 2}\right)}}\left\langle-N_{N}\left(\hat{\phi}^{n+1 / 2}\right), \phi^{n+1}-\phi^{n}\right\rangle, \\
\left(r^{n+1}-r^{n}\right)\left(r^{n+1}+r^{n}\right)= & \frac{r^{n+1 / 2}}{\sqrt{E_{1, N}\left(\hat{\phi}^{n+1 / 2}\right)}}\left\langle N_{N}\left(\hat{\phi}^{n+1 / 2}\right), \phi^{n+1}-\phi^{n}\right\rangle .
\end{aligned}
$$

In turn, by adding (2.37a), (2.37b) and (2.37c), we obtain

$$
\left\langle L_{N}\left(\frac{3}{4} \phi^{n+1}+\frac{1}{4} \phi^{n-1}\right), \phi^{n+1}-\phi^{n}\right\rangle+\left\langle r^{n+1}-r^{n}, 2 r^{n+1}\right\rangle=-\Delta t\left\|\mu_{N}^{n+1 / 2}\right\|_{2}^{2} .
$$


Meanwhile, the derivation of the following two identities are straightforward:

$$
\begin{aligned}
& \left\langle L_{N}\left(\frac{3}{4} \phi^{n+1}+\frac{1}{4} \phi^{n-1}\right), \phi^{n+1}-\phi^{n}\right\rangle \\
& =\left\langle L_{N}^{\frac{1}{2}}\left(\frac{3}{4} \phi^{n+1}+\frac{1}{4} \phi^{n-1}\right), L_{N}^{\frac{1}{2}}\left(\phi^{n+1}-\phi^{n}\right)\right\rangle \\
& =\frac{1}{2}\left(\left\|L_{N}^{\frac{1}{2}} \phi^{n+1}\right\|_{2}^{2}-\left\|L_{N}^{\frac{1}{2}} \phi^{n}\right\|_{2}^{2}\right)+\frac{1}{8}\left(\left\|L_{N}^{\frac{1}{2}}\left(\phi^{n+1}-\phi^{n}\right)\right\|_{2}^{2}-\left\|L_{N}^{\frac{1}{2}}\left(\phi^{n}-\phi^{n-1}\right)\right\|_{2}^{2}\right) \\
& \quad+\frac{1}{8}\left(\| L_{N}^{\frac{1}{2}}\left(\phi^{n+1}-2 \phi^{n}+\phi^{n-1} \|_{2}^{2}\right),\right. \\
& \left(r^{n+1}-r^{n}\right)\left(r^{n+1}+r^{n}\right)=\left|r^{n+1}\right|^{2}-\left|r^{n}\right|^{2},
\end{aligned}
$$

in which identity (2.13) has been applied in the first step of (2.39a). Going back (2.38), we arrive at

$$
\begin{aligned}
& \mathcal{E}_{N}\left(\phi^{n+1}, \phi^{n}, r^{n+1}\right)-\mathcal{E}_{N}\left(\phi^{n}, \phi^{n-1}, r^{n}\right) \\
= & -\frac{1}{8}\left\|L_{N}^{\frac{1}{2}}\left(\phi^{n+1}-2 \phi^{n}+\phi^{n-1}\right)\right\|_{2}^{2}-\Delta t\left\|\mu_{N}^{n+1 / 2}\right\|_{2}^{2} \leq 0 .
\end{aligned}
$$

This completes the proof of Theorem 2.2.

As a direct consequence of the energy stability, a uniform in time $H_{N}^{2}$ bound for the numerical solution is derived as follows.

Corollary 2.1. Suppose that the initial data are sufficiently regular so that

$$
\frac{1}{2}\left\|L_{N}^{\frac{1}{2}} \phi^{0}\right\|_{2}^{2}+\left|r^{0}\right|^{2}+\frac{1}{8}\left\|L_{N}^{\frac{1}{2}}\left(\phi^{0}-\phi^{-1}\right)\right\|_{2}^{2} \leq \tilde{C}_{0}
$$

for some $\tilde{C}_{0}$ that is independent of $h$. Then we have the following uniform (in time) $H_{N}^{2}$ bound for the numerical solution:

$$
\left\|\phi_{S}^{m}\right\|_{H^{2}} \leq \tilde{C}_{1}, \quad \forall m \geq 1,
$$

in which $\phi_{S}^{m}$ stands for the spectral interpolation of the numerical solution $\phi^{m}$, as given by formula (2.8). The constant $\tilde{C}_{1}>0$ depends on $\Omega$ and $\tilde{C}_{0}$, but is independent of $h, \Delta t$ and final time.

Proof. As a result of (2.35), the following energy bound is available:

$$
\begin{aligned}
& \frac{1}{2}\left\|L_{N}^{\frac{1}{2}} \phi^{m}\right\|_{2}^{2} \leq \mathcal{E}_{N}\left(\phi^{m}, \phi^{m-1}, r^{m}\right) \leq \mathcal{E}_{N}\left(\phi^{0}, \phi^{-1}, r^{0}\right) \\
= & \frac{1}{2}\left\|L_{N}^{\frac{1}{2}} \phi^{0}\right\|_{2}^{2}+\left|r^{0}\right|^{2}+\frac{1}{8}\left\|L_{N}^{\frac{1}{2}}\left(\phi^{0}-\phi^{-1}\right)\right\|_{2}^{2} \leq \tilde{C}_{0}
\end{aligned}
$$

for any $m \geq 1$. On the other hand, since $L_{N}^{\frac{1}{2}} f=-\varepsilon \Delta_{N} f$, for any $f \in \mathcal{G}_{N}$, we see that

$$
\frac{\varepsilon^{2}}{2}\left\|\Delta_{N} \phi^{m}\right\|_{2}^{2} \leq \tilde{C}_{0}, \quad \text { so that }\left\|\Delta_{N} \phi^{m}\right\|_{2} \leq \sqrt{2} \varepsilon^{-2} \tilde{C}_{0}^{\frac{1}{2}}, \quad \forall m \geq 1 .
$$


This in turn leads to

$$
\left\|\Delta \phi_{S}^{m}\right\|=\left\|\Delta_{N} \phi^{m}\right\|_{2} \leq \sqrt{2} \varepsilon^{-2} \tilde{C}_{0}^{\frac{1}{2}}, \quad \text { since } \phi^{m} \in \mathcal{G}_{N} .
$$

Meanwhile, by the mass conservative property stated in Theorem 2.1, we have

$$
\int_{\Omega} \phi_{S}^{m} d \mathbf{x}=\overline{\phi^{m}}=\beta_{0}, \quad \forall m \geq 0,
$$

in which the first step is based on the fact that $\phi^{m} \in \mathcal{G}_{N}$. In turn, an application of elliptic regularity implies that

$$
\left\|\phi_{S}^{m}\right\|_{H^{2}} \leq C\left(\left|\int_{\Omega} \phi_{S}^{m} d \mathbf{x}\right|+\left\|\Delta \phi_{S}^{m}\right\|\right) \leq C\left(\left|\beta_{0}\right|+\sqrt{2} \varepsilon^{-2} \tilde{C}_{0}^{\frac{1}{2}}\right):=\tilde{C}_{1}, \quad \forall m \geq 1 .
$$

This completes the proof of Corollary 2.1.

Remark 2.1. It is obvious that the modified energy functional (2.34) is the second order approximation to the original discrete energy (2.16), under certain regularity assumption for the numerical solution. Meanwhile, such a modified discrete energy is in terms of a scalar auxiliary variable $r$, combined with the linear surface diffusion energy part, not fully in terms of the original phase variable $\phi$, as formulated in (2.16). Although a direct bound of the original energy functional is not available in terms of the initial data, a uniform in time $H_{N}^{2}$ bound for the numerical solution could be derived, up to a constant multiple, as demonstrated in Corollary 2.1.

Remark 2.2. As a combination of the uniform in time $H_{N}^{2}$ bound (2.41) and the discrete Sobolev embedding inequality (2.17), we arrive at a uniform in time $W_{N}^{1,6}$ estimate for the numerical solution:

$$
\left\|\nabla_{N} \phi^{m}\right\|_{6} \leq C \tilde{C}_{1}, \quad \forall m \geq 1 .
$$

And also, the modified energy inequality (2.42) indicates that

$$
\left|r^{m}\right|^{2} \leq \tilde{C}_{0}, \quad \text { so that } r^{m} \leq \tilde{C}_{0}^{\frac{1}{2}}, \quad \forall m \geq 1 .
$$

These estimates will be useful in the higher order stability analysis presented below.

\section{A uniform-in-time $H^{3}$ estimate for the numerical solution}

Theorem 3.1. For the numerical solution (2.24), the following estimate is available:

$$
\left\|\phi_{S}^{m}\right\|_{H^{3}} \leq Q^{(3)}, \quad \forall m \geq 1,
$$

in which $\phi_{S}^{m}$ stands for the spectral interpolation of the numerical solution $\phi^{m}$, as given by formula (2.8). The constant $Q^{(3)}$ only depends on the initial $H^{3}$ data and the domain, and it is independent of $\Delta t, h$ and $T$. 
Proof. Taking a discrete inner product with (2.24a) by $-2 \Delta_{N}^{3} \phi^{n+1}$, we obtain

$$
\begin{aligned}
& \frac{1}{\Delta t}\left\langle\phi^{n+1}-\phi^{n},-2 \Delta_{N}^{3} \phi^{n+1}\right\rangle-\varepsilon^{2}\left\langle\Delta_{N}^{3} \phi^{n+1}, \Delta_{N}^{2}\left(\frac{3}{2} \phi^{n+1}+\frac{1}{2} \phi^{n-1}\right)\right\rangle \\
= & 2 \frac{r^{n+1 / 2}}{\sqrt{E_{1, N}\left(\hat{\phi}^{n+1 / 2}\right)}}\left\langle N_{N}\left(\hat{\phi}^{n+1 / 2}\right), \Delta_{N}^{3} \phi^{n+1}\right\rangle .
\end{aligned}
$$

The temporal stencil term could be handled in a straightforward way:

$$
\begin{aligned}
& \left\langle\phi^{n+1}-\phi^{n},-2 \Delta_{N}^{3} \phi^{n+1}\right\rangle \\
= & 2\left\langle\nabla_{N} \Delta_{N}\left(\phi^{n+1}-\phi^{n}\right), \nabla_{N} \Delta_{N} \phi^{n+1}\right\rangle \\
= & \left\|\nabla_{N} \Delta_{N} \phi^{n+1}\right\|_{2}^{2}-\left\|\nabla_{N} \Delta_{N} \phi^{n}\right\|_{2}^{2}+\left\|\nabla_{N} \Delta_{N}\left(\phi^{n+1}-2 \phi^{n}+\phi^{n-1}\right)\right\|_{2}^{2} .
\end{aligned}
$$

The surface diffusion part could be analyzed as follows

$$
\begin{aligned}
& -\left\langle\Delta_{N}^{3} \phi^{n+1}, \Delta_{N}^{2}\left(\frac{3}{2} \phi^{n+1}+\frac{1}{2} \phi^{n-1}\right)\right\rangle \\
= & \left\langle\nabla_{N} \Delta_{N}^{2} \phi^{n+1}, \nabla_{N} \Delta_{N}^{2}\left(\frac{3}{2} \phi^{n+1}+\frac{1}{2} \phi^{n-1}\right)\right\rangle \\
\geq & \frac{3}{2}\left\|\nabla_{N} \Delta_{N}^{2} \phi^{n+1}\right\|_{2}^{2}-\frac{1}{4}\left(\left\|\nabla_{N} \Delta_{N}^{2} \phi^{n+1}\right\|_{2}^{2}+\left\|\nabla_{N} \Delta_{N}^{2} \phi^{n-1}\right\|_{2}^{2}\right) \\
\geq & \frac{5}{4}\left\|\nabla_{N} \Delta_{N}^{2} \phi^{n+1}\right\|_{2}^{2}-\frac{1}{4}\left\|\nabla_{N} \Delta_{N}^{2} \phi^{n-1}\right\|_{2}^{2} .
\end{aligned}
$$

For the right hand side nonlinear inner product, we begin with the following observations:

$$
E_{1, N}\left(\hat{\phi}^{n+1 / 2}\right) \geq|\Omega|, \quad\left|r^{n+1 / 2}\right| \leq \tilde{C}_{0}^{\frac{1}{2}} \quad(\text { by }(2.48)) .
$$

These two bounds imply that

$$
\frac{r^{n+1 / 2}}{\sqrt{E_{1, N}\left(\hat{\phi}^{n+1}\right)}} \leq\left(\frac{\tilde{C}_{0}}{|\Omega|}\right)^{\frac{1}{2}} .
$$

Meanwhile, the following summation by parts formula is applied

$$
\begin{aligned}
& 2 \frac{r^{n+1 / 2}}{\sqrt{E_{1, N}\left(\hat{\phi}^{n+1 / 2}\right)}}\left\langle N_{N}\left(\hat{\phi}^{n+1 / 2}\right), \Delta_{N}^{3} \phi^{n+1}\right\rangle \\
= & -2 \frac{r^{n+1 / 2}}{\sqrt{E_{1, N}\left(\hat{\phi}^{n+1 / 2}\right)}}\left\langle\nabla_{N} N_{N}\left(\hat{\phi}^{n+1 / 2}\right), \nabla_{N} \Delta_{N}^{2} \phi^{n+1}\right\rangle .
\end{aligned}
$$


For the nonlinear term, the following expansion is recalled

$$
\nabla_{N} N_{N}\left(\hat{\phi}^{n+1}\right)=-\nabla_{N} \nabla_{N} \cdot\left(\left|\nabla_{N} \hat{\phi}^{n+1}\right|^{2} \nabla_{N} \hat{\phi}^{n+1}\right)+\nabla_{N} \Delta_{N} \hat{\phi}^{n+1} \text {. }
$$

The linear part could be controlled in a standard fashion:

$$
\begin{aligned}
& -2 \frac{r^{n+1 / 2}}{\sqrt{E_{1, N}\left(\hat{\phi}^{n+1 / 2}\right)}}\left\langle\nabla_{N} \Delta_{N} \hat{\phi}^{n+1 / 2}, \nabla_{N} \Delta_{N}^{2} \phi^{n+1}\right\rangle \\
\leq & 2\left(\frac{\tilde{C}_{0}}{|\Omega|}\right)^{\frac{1}{2}}\left\|\nabla_{N} \Delta_{N} \hat{\phi}^{n+1 / 2}\right\|_{2} \cdot\left\|\nabla_{N} \Delta_{N}^{2} \phi^{n+1}\right\|_{2} \\
\leq & \frac{4 \tilde{C}_{0} \varepsilon^{-2}}{|\Omega|}\left\|\nabla_{N} \Delta_{N} \hat{\phi}^{n+1 / 2}\right\|_{2}^{2}+\frac{1}{4} \varepsilon^{2}\left\|\nabla_{N} \Delta_{N}^{2} \phi^{n+1}\right\|_{2}^{2} .
\end{aligned}
$$

For the nonlinear 4-Laplacian part, the following grid function is introduced:

$$
\hat{q}^{n+1 / 2}:=\left|\nabla_{N} \hat{\phi}^{n+1 / 2}\right|^{2} \nabla_{N} \hat{\phi}^{n+1 / 2} \text {. }
$$

This in turn implies that

$$
\left\|\nabla_{N} \nabla_{N} \cdot\left(\left|\nabla_{N} \hat{\phi}^{n+1 / 2}\right|^{2} \nabla_{N} \hat{\phi}^{n+1 / 2}\right)\right\|_{2}=\left\|\nabla\left(\nabla \cdot \hat{q}_{S}^{n+1 / 2}\right)\right\|_{L^{2}}
$$

in which $\hat{q}_{S}^{n+1 / 2}$ is the spectral interpolation of $\hat{q}^{n+1 / 2}$, given by formula (2.8). Moreover, since $\hat{q}^{n+1 / 2}$ is the point-wise interpolation of the continuous function

$$
\varphi_{\hat{q}^{n+1 / 2}}:=\left|\nabla \hat{\phi}_{S}^{n+1 / 2}\right|^{2} \nabla \hat{\phi}_{S}^{n+1 / 2} \quad \text { with } \hat{\phi}_{S}^{n+1 / 2}=\frac{3}{2} \phi_{S}^{n}-\frac{1}{2} \phi_{S}^{n-1},
$$

we see that $\hat{q}_{S}^{n+1 / 2}=R_{N}\left(\varphi_{\hat{q}^{n+1 / 2}}\right)$. In turn, by making use of the aliasing error control inequality stated in Lemma 2.1, we conclude that

$$
\left\|\nabla\left(\nabla \cdot \hat{q}_{S}^{n+1 / 2}\right)\right\|_{L^{2}} \leq\left\|\hat{q}_{S}^{n+1 / 2}\right\|_{H^{2}}=\left\|R_{N}\left(\varphi_{\hat{q}^{n+1 / 2}}\right)\right\|_{H^{2}} \leq 3\left\|\varphi_{\hat{q}^{n+1 / 2}}\right\|_{H^{2}}
$$

since $\varphi_{\hat{q}^{n+1 / 2}} \in \mathcal{P}_{3 K}$. Meanwhile, for $\varphi_{\hat{q}^{n+1 / 2}}$ given by (3.12), a detailed expansion and repeated applications of Hölder inequality indicate that

$$
\begin{aligned}
\left\|\varphi_{\hat{q}^{n+1 / 2}}\right\|_{H^{2}} & \leq C\left(\left\|\varphi_{\hat{q}^{n+1 / 2}}\right\|+\left\|\Delta \varphi_{\hat{q}^{n+1 / 2}}\right\|\right) \\
& =C\left(\left\|\left|\nabla \hat{\phi}_{S}^{n+1 / 2}\right|^{2} \nabla \hat{\phi}_{S}^{n+1 / 2}\right\|+\left\|\Delta\left(\left|\nabla \hat{\phi}_{S}^{n+1 / 2}\right|^{2} \nabla \hat{\phi}_{S}^{n+1 / 2}\right)\right\|\right) \\
& \leq C\left(\left\|\nabla \hat{\phi}_{S}^{n+1}\right\|_{L^{\infty}}^{2} \cdot\left\|\nabla \hat{\phi}_{S}^{n+1}\right\|_{H^{2}}+\left\|\nabla \nabla \hat{\phi}_{S}^{n+1}\right\|_{L^{6}}^{2} \cdot\left\|\nabla \hat{\phi}_{S}^{n+1}\right\|_{L^{6}}\right) .
\end{aligned}
$$


Furthermore, the following 2-D Sobolev embedding and interpolation inequalities could be derived:

$$
\begin{aligned}
&\left\|\nabla \hat{\phi}_{S}^{n+1 / 2}\right\|_{L^{\infty}} \leq C\left(\left\|\Delta \hat{\phi}_{S}^{n+1 / 2}\right\|+\left\|\Delta \hat{\phi}_{S}^{n+1 / 2}\right\|^{\frac{5}{6}} \cdot\left\|\nabla \Delta^{2} \hat{\phi}_{S}^{n+1 / 2}\right\|^{\frac{1}{6}}\right) \\
& \leq C\left(\tilde{C}_{1}+\tilde{C}_{1}^{\frac{5}{6}}\left\|\Delta^{3} \hat{\phi}_{S}^{n+1 / 2}\right\|^{\frac{1}{6}}\right) \\
&\left\|\nabla \hat{\phi}_{S}^{n+1 / 2}\right\|_{L^{6}} \leq C\left\|\Delta \hat{\phi}_{S}^{n+1 / 2}\right\| \leq C \tilde{C}_{1} \\
&\left\|\nabla \nabla \hat{\phi}_{S}^{n+1 / 2}\right\|_{L^{6}} \leq C\left\|\nabla \nabla \hat{\phi}_{S}^{n+1 / 2}\right\|_{H^{1}} \leq C\left\|\Delta \hat{\phi}_{S}^{n+1 / 2}\right\|^{\frac{2}{3}} \cdot\left\|\nabla \Delta^{2} \hat{\phi}_{S}^{n+1 / 2}\right\|^{\frac{1}{3}} \\
& \leq C \tilde{C}_{1}^{\frac{2}{3}}\left\|\nabla \Delta^{2} \hat{\phi}_{S}^{n+1 / 2}\right\|^{\frac{1}{3}} \\
&\left\|\nabla \hat{\phi}_{S}^{n+1 / 2}\right\|_{H^{2}} \leq C\left\|\Delta \hat{\phi}_{S}^{n+1 / 2}\right\|^{\frac{2}{3}} \cdot\left\|\Delta^{3} \hat{\phi}_{S}^{n+1 / 2}\right\|^{\frac{1}{3}} \leq C \tilde{C}_{1}^{\frac{2}{3}}\left\|\nabla \Delta^{2} \hat{\phi}_{S}^{n+1 / 2}\right\|^{\frac{1}{3}},
\end{aligned}
$$

in which the uniform in time $H^{2}$ bound (2.41) of the numerical solution has been extensively used. In turn, a substitution of the above estimates into (3.14) yields

$$
\left\|\varphi_{\hat{q}^{n+1}}\right\|_{H^{3}} \leq C\left(\tilde{C}_{1}^{3}+\tilde{C}_{1}^{\frac{7}{3}}\left\|\Delta^{3} \hat{\phi}_{S}^{n+1 / 2}\right\|^{\frac{2}{3}}\right) .
$$

Subsequently, its combination with (3.11) and (3.13) reveals that

$$
\begin{aligned}
& \left\|\nabla_{N} \nabla_{N} \cdot\left(\left|\nabla_{N} \hat{\phi}^{n+1 / 2}\right|^{2} \nabla_{N} \hat{\phi}^{n+1 / 2}\right)\right\|_{2} \\
\leq & C\left(\tilde{C}_{1}^{3}+\tilde{C}_{1}^{\frac{7}{3}}\left\|\nabla \Delta^{2} \hat{\phi}_{S}^{n+1 / 2}\right\|^{\frac{2}{3}}\right) \\
\leq & C\left(\tilde{C}_{1}^{3}+\tilde{C}_{1}^{\frac{7}{3}}\left\|\nabla_{N} \Delta_{N}^{2} \hat{\phi}^{n+1 / 2}\right\|^{\frac{2}{3}}\right),
\end{aligned}
$$

in which the fact that $\hat{\phi}_{S}^{n+1 / 2} \in \mathcal{P}_{K}$ has been applied in the last step. As a consequence, we arrive at

$$
\begin{aligned}
& -2 \frac{r^{n+1 / 2}}{\sqrt{E_{1, N}\left(\hat{\phi}^{n+1 / 2}\right)}}\left\langle\nabla_{N} \nabla_{N} \cdot\left(\left|\nabla_{N} \hat{\phi}^{n+1 / 2}\right|^{2} \nabla_{N} \hat{\phi}^{n+1 / 2}\right), \nabla_{N} \Delta_{N}^{2} \phi^{n+1}\right\rangle \\
\leq & 2\left(\frac{\tilde{C}_{0}}{|\Omega|}\right)^{\frac{1}{2}}\left\|\nabla_{N} \nabla_{N} \cdot\left(\left|\nabla_{N} \hat{\phi}^{n+1 / 2}\right|^{2} \nabla_{N} \hat{\phi}^{n+1 / 2}\right)\right\|_{2} \cdot\left\|\nabla_{N} \Delta_{N}^{2} \phi^{n+1}\right\|_{2} \\
\leq & C\left(\tilde{C}_{1}^{3}+\tilde{C}_{1}^{\frac{7}{3}}\left\|\Delta_{N}^{3} \hat{\phi}^{n+1 / 2}\right\|^{\frac{2}{3}}\right) \cdot\left\|\nabla_{N} \Delta_{N}^{2} \phi^{n+1}\right\|_{2} \\
\leq & C \varepsilon^{-2}\left(\tilde{C}_{1}^{6}+\tilde{C}_{1}^{\frac{14}{3}}\left\|\nabla_{N} \Delta_{N}^{2} \hat{\phi}^{n+1 / 2}\right\|^{\frac{4}{3}}\right)+\frac{\varepsilon^{2}}{4}\left\|\nabla_{N} \Delta_{N}^{2} \phi^{n+1}\right\|_{2}^{2} .
\end{aligned}
$$

A combination of (3.9) and (3.18) leads to

$$
2 \frac{r^{n+1 / 2}}{\sqrt{E_{1, N}\left(\hat{\phi}^{n+1}\right)}}\left\langle N_{N}\left(\hat{\phi}^{n+1}\right), \Delta_{N}^{3} \phi^{n+1}\right\rangle
$$




$$
\begin{aligned}
& \leq \frac{4 \tilde{C}_{0} \varepsilon^{-2}}{|\Omega|}\left\|\nabla_{N} \Delta_{N} \hat{\phi}^{n+1 / 2}\right\|_{2}^{2}+C \varepsilon^{-2}\left(\tilde{C}_{1}^{6}+\tilde{C}_{1}^{\frac{14}{3}}\left\|\nabla_{N} \Delta_{N}^{2} \hat{\phi}^{n+1 / 2}\right\|^{\frac{4}{3}}\right) \\
& \quad+\frac{\varepsilon^{2}}{2}\left\|\nabla_{N} \Delta_{N}^{2} \phi^{n+1}\right\|_{2}^{2} .
\end{aligned}
$$

Finally, a substitution of (3.3), (3.4) and (3.19) into (3.2) results in

$$
\begin{aligned}
& \frac{1}{\Delta t}\left(\left\|\nabla_{N} \Delta_{N} \phi^{n+1}\right\|_{2}^{2}-\left\|\nabla_{N} \Delta_{N} \phi^{n}\right\|_{2}^{2}+\left\|\nabla_{N} \Delta_{N}\left(\phi^{n+1}-2 \phi^{n}+\phi^{n-1}\right)\right\|_{2}^{2}\right) \\
& +\frac{3}{4} \varepsilon^{2}\left\|\nabla_{N} \Delta_{N}^{2} \phi^{n+1}\right\|_{2}^{2}-\frac{1}{4} \varepsilon^{2}\left\|\nabla_{N} \Delta_{N}^{2} \phi^{n-1}\right\|_{2}^{2} \\
\leq & \frac{4 \tilde{C}_{0} \varepsilon^{-2}}{|\Omega|}\left\|\nabla_{N} \Delta_{N} \hat{\phi}^{n+1 / 2}\right\|_{2}^{2}+C \varepsilon^{-2}\left(\tilde{C}_{1}^{6}+\tilde{C}_{1}^{\frac{14}{3}}\left\|\nabla_{N} \Delta_{N}^{2} \hat{\phi}^{n+1 / 2}\right\|^{\frac{4}{3}}\right) .
\end{aligned}
$$

Meanwhile, the following interpolation inequality and Cauchy inequality are available:

$$
\begin{aligned}
& \left\|\nabla_{N} \Delta_{N} \hat{\phi}^{n+1 / 2}\right\|_{2} \leq\left\|\Delta_{N} \hat{\phi}^{n+1 / 2}\right\|_{2}^{\frac{2}{3}} \cdot\left\|\nabla_{N} \Delta_{N}^{2} \hat{\phi}^{n+1}\right\|_{2}^{\frac{1}{3}} \leq\left(2 \tilde{C}_{1}\right)^{\frac{2}{3}}\left\|\nabla_{N} \Delta_{N}^{2} \hat{\phi}^{n+1 / 2}\right\|_{2}^{\frac{1}{3}}, \\
& \left\|\nabla_{N} \Delta_{N}^{2} \hat{\phi}^{n+1 / 2}\right\|_{2}^{2}=\left\|\nabla_{N} \Delta_{N}^{2}\left(\frac{3}{2} \phi^{n}-\frac{1}{2} \phi^{n-1}\right)\right\|_{2}^{2} \leq 3\left\|\nabla_{N} \Delta_{N}^{2} \phi^{n}\right\|_{2}^{2}+\left\|\nabla_{N} \Delta_{N}^{2} \phi^{n-1}\right\|_{2}^{2}
\end{aligned}
$$

Then we obtain the following estimates:

$$
\begin{aligned}
& \frac{4 \tilde{C}_{0} \varepsilon^{-2}}{|\Omega|}\left\|\nabla_{N} \Delta_{N} \hat{\phi}^{n+1 / 2}\right\|_{2}^{2} \\
& \leq \frac{2^{\frac{10}{3}} \tilde{C}_{0} \tilde{C}_{1}^{\frac{4}{3}} \varepsilon^{-2}}{|\Omega|}\left\|\nabla_{N} \Delta_{N}^{2} \hat{\phi}^{n+1 / 2}\right\|_{2}^{\frac{2}{3}} \\
& \leq \frac{C \tilde{C}_{0}^{\frac{3}{2}} \tilde{C}_{1}^{2} \varepsilon^{-4}}{|\Omega|^{\frac{3}{2}}}+\frac{\varepsilon^{2}}{32}\left\|\nabla_{N} \Delta_{N}^{2} \hat{\phi}^{n+1 / 2}\right\|_{2}^{2} \\
& \leq \frac{C \tilde{C}_{0}^{\frac{3}{2}} \tilde{C}_{1}^{2} \varepsilon^{-4}}{|\Omega|^{\frac{3}{2}}}+\frac{\varepsilon^{2}}{32}\left(3\left\|\nabla_{N} \Delta_{N}^{2} \phi^{n}\right\|_{2}^{2}+\left\|\nabla_{N} \Delta_{N}^{2} \phi^{n-1}\right\|_{2}^{2}\right) \\
& C \tilde{C}_{1}^{\frac{14}{3}} \varepsilon^{-2}\left\|\nabla_{N} \Delta_{N}^{2} \hat{\phi}^{n+1 / 2}\right\|^{\frac{4}{3}} \\
& \leq C \tilde{C}_{1}^{14} \varepsilon^{-10}+\frac{\varepsilon^{2}}{32}\left\|\nabla_{N} \Delta_{N}^{2} \hat{\phi}^{n+1 / 2}\right\|^{2} \\
& \leq C \tilde{C}_{1}^{14} \varepsilon^{-10}+\frac{\varepsilon^{2}}{32}\left(3\left\|\nabla_{N} \Delta_{N}^{2} \phi^{n}\right\|_{2}^{2}+\left\|\nabla_{N} \Delta_{N}^{2} \phi^{n-1}\right\|_{2}^{2}\right),
\end{aligned}
$$

in which the Young's inequality has been applied in the first step of (3.22b). Going back (3.20), we arrive at

$$
\begin{aligned}
& \frac{1}{\Delta t}\left(\left\|\nabla_{N} \Delta_{N} \phi^{n+1}\right\|_{2}^{2}-\left\|\nabla_{N} \Delta_{N} \phi^{n}\right\|_{2}^{2}\right)+\frac{3}{4} \varepsilon^{2}\left\|\nabla_{N} \Delta_{N}^{2} \phi^{n+1}\right\|_{2}^{2}-\frac{1}{4} \varepsilon^{2}\left\|\nabla_{N} \Delta_{N}^{2} \phi^{n-1}\right\|_{2}^{2} \\
\leq & \frac{\varepsilon^{2}}{16}\left(3\left\|\nabla_{N} \Delta_{N}^{2} \phi^{n}\right\|_{2}^{2}+\left\|\nabla_{N} \Delta_{N}^{2} \phi^{n-1}\right\|_{2}^{2}\right)+\frac{C \tilde{C}_{0}^{\frac{3}{2}} \tilde{C}_{1}^{2} \varepsilon^{-4}}{|\Omega|^{\frac{3}{2}}}+C \varepsilon^{-10}\left(\tilde{C}_{1}^{14}+1\right) .
\end{aligned}
$$


Moreover, the following quantity is introduced:

$$
G^{n+1}:=\left\|\nabla_{N} \Delta_{N} \phi^{n+1}\right\|_{2}^{2}+\frac{9 \varepsilon^{2}}{16} \Delta t\left\|\nabla_{N} \Delta_{N}^{2} \phi^{n+1}\right\|_{2}^{2}+\frac{5 \varepsilon^{2}}{16} \Delta t\left\|\nabla_{N} \Delta_{N}^{2} \phi^{n}\right\|_{2}^{2} .
$$

By adding $\frac{3}{8} \varepsilon^{2}\left\|\nabla_{N} \Delta_{N}^{3} \phi^{n}\right\|_{2}^{2}$ on both sides of (3.23), we obtain the following inequality:

$$
G^{n+1}-G^{n}+\frac{3 \varepsilon^{2}}{16} \Delta t\left\|\nabla_{N} \Delta_{N}^{2} \phi^{n+1}\right\|_{2}^{2}+\frac{\varepsilon^{2}}{16} \Delta t\left\|\nabla_{N} \Delta_{N}^{2} \phi^{n}\right\|_{2}^{2} \leq M^{(0)} \Delta t
$$

with

$$
M^{(0)}=\frac{C \tilde{C}_{0}^{\frac{3}{2}} \tilde{C}_{1}^{2} \varepsilon^{-4}}{|\Omega|^{\frac{3}{2}}}+C \varepsilon^{-10}\left(\tilde{C}_{1}^{14}+1\right) .
$$

In addition, the following elliptic regularity estimates are valid:

$$
C_{2}\left\|\nabla_{N} \Delta_{N} \phi^{n+1}\right\|_{2}^{2} \leq\left\|\nabla_{N} \Delta_{N}^{2} \phi^{n+1}\right\|_{2}^{2}, \quad C_{2}\left\|\nabla_{N} \Delta_{N} \phi^{n}\right\|_{2}^{2} \leq\left\|\nabla_{N} \Delta_{N}^{2} \phi^{n}\right\|_{2}^{2},
$$

so that we arrive at

$$
\frac{1}{8} C_{2} G^{n+1} \leq \frac{3}{16}\left\|\nabla_{N} \Delta_{N}^{2} \phi^{n+1}\right\|_{2}^{2}+\frac{1}{16}\left\|\nabla_{N} \Delta_{N}^{2} \phi^{n}\right\|_{2}^{2} .
$$

Going back (3.25), we get

$$
G^{n+1}-G^{n}+\frac{C_{2} \varepsilon^{2}}{8} \Delta t G^{n+1} \leq M^{(0)} \Delta t .
$$

An application of induction argument implies that

$$
G^{n+1} \leq\left(1+\frac{C_{2} \varepsilon^{2}}{8} \Delta t\right)^{-(n+1)} G^{0}+\frac{8 M^{(0)}}{C_{2} \varepsilon^{2}} .
$$

Of course, we could introduce a uniform in time quantity

$$
B_{3}^{*}:=G^{0}+\frac{8 M^{(0)}}{C_{2} \varepsilon^{2}}
$$

so that

$$
\left\|\nabla_{N} \Delta_{N} \phi^{m}\right\|^{2} \leq G^{m} \leq B_{3}^{*}
$$

for any $m \geq 0$. In turn, an application of elliptic regularity shows that

$$
\left\|\phi_{S}^{m}\right\|_{H^{3}} \leq C\left(\left|\overline{\phi^{m}}\right|+\left\|\nabla \Delta \phi^{m}\right\|\right) \leq C\left(\left|\beta_{0}\right|+\left(B_{3}^{*}\right)^{1 / 2}\right):=Q^{(3)}, \quad \forall m \geq 0,
$$

in which the uniform in time constant $Q^{(3)}$ depends on $\Omega$ and the initial $H^{3}$ data. This finishes the proof of Theorem 3.1. 
Remark 3.1. Higher order $H^{m}$ estimate (beyond the norm given by the physical energy) is available for many gradient flows, due to the analytic property of the surface diffusion parabolic operator; see the related discussions in [4]. There have also been quite a few works of uniform in time $H^{2}$ estimate for certain energy stable numerical schemes for the Cahn-Hilliard equation $[16,32,56]$, beyond the $H^{1}$ bound given by the energy estimate. In fact, similar estimates have also been reported for 2-D incompressible Navier-Stokes equations, in terms of the first, second and higher order temporal numerical approximations; see the delated works $[14,28,62]$, etc.

Remark 3.2. As can be observed in the analysis (3.4) for the surface diffusion term, the alternate temporal stencil structure, which gives $3 / 4$ and $1 / 4$ coefficient distribution at time steps $t^{n+1}, t^{n-1}$, respectively, plays an important role to pass through the nonlinear estimate. Because of the higher concentration at time step $t^{n+1}$, the additional diffusion inner product term is able to control the corresponding nonlinear growth given by (3.19), which turns out to be an essential point in the $H^{3}$ estimate for the numerical solution. Instead, if the standard Crank-Nicolson approximation is applied, such an additional diffusion inner product term would not be available in the derivation in (3.19), so that the nonlinear growth in (3.19) could hardly be controlled. As a result, the standard CrankNicolson method for the surface diffusion term is able to preserve the modified energy stability, in a similar way as in Theorem 2.2, while a theoretical justification of the $H^{3}$ estimate for the numerical solution would not go through.

\section{The optimal rate convergence analysis}

Now we proceed into the convergence analysis for the numerical scheme (2.24). Due to the SAV structure of the algorithm, the error estimate has to be performed in the energy norm, i.e., in the $\ell^{\infty}\left(0, T ; H_{N}^{2}\right) \cap \ell^{2}\left(0, T ; H_{N}^{4}\right)$ for the phase variable. Similar techniques have also been applied to the convergence estimate [45] for the SAV scheme applied to CahnHilliard equation. These ideas have also been reported for the corresponding analysis for the phase field flow coupled with fluid motion $[6,9,18,19,47]$. With an initial data with sufficient regularity, we could assume that the exact solution has regularity of class $\mathcal{R}$ :

$$
\Phi \in \mathcal{R}:=H^{3}\left(0, T ; C^{0}\right) \cap H^{2}\left(0, T ; H^{4}\right) \cap L^{\infty}\left(0, T ; H^{m+4}\right) .
$$

In particular, the following bound is available for the exact solution:

$$
\left\|\partial_{t}^{m} \Phi\right\|_{L^{\infty}\left(0, T ; L^{\infty}\right)} \leq C^{*}, \quad(1 \leq m \leq 3), \quad\left\|\Phi^{k}\right\|_{H^{m+4}} \leq C^{*}, \quad \forall k \geq 0 .
$$

Theorem 4.1. Given initial data $\Phi_{0} \in H_{\mathrm{per}}^{m+4}(\Omega)$, suppose the exact solution for SS equation (1.3) is of regularity class $\mathcal{R}$. For $\Delta t$ and $h$ are sufficiently small, we have

$$
\max _{0 \leq n \leq M}\left\|\Delta_{N}\left(\Phi^{n}-\phi^{n}\right)\right\|_{2}+\left(\Delta t \sum_{k=1}^{M}\left\|\Delta_{N}^{2}\left(\Phi^{k}-\phi^{k}\right)\right\|_{2}^{2}\right)^{1 / 2} \leq C\left(\Delta t^{2}+h^{m}\right),
$$

where $C>0$ is independent of $\Delta t$ and $h$, and $\Delta t=T / M$. 


\subsection{The consistency analysis}

For $\Phi \in \mathcal{R}$, we construct an approximate scalar value of $R$ as follows

$$
\begin{aligned}
& R^{n+1}:=\sqrt{E_{1, N}\left(\Phi^{n+1}\right)}, \\
& E_{1, N}^{n+1}\left(\Phi^{n+1}\right)=\frac{1}{4}\left\|\nabla_{N} \Phi^{n+1}\right\|_{4}^{4}-\frac{1}{2}\left\|\nabla_{N} \Phi^{n+1}\right\|_{2}^{2}+\frac{5}{4}|\Omega|, \\
& R^{n+1 / 2}=\frac{1}{2}\left(R^{n+1}+R^{n}\right) .
\end{aligned}
$$

A similar extrapolation

$$
\hat{\Phi}^{n+1}:=2 \Phi^{n}-\Phi^{n-1}
$$

is taken. In turn, a careful consistency analysis indicates the following truncation error estimate:

$$
\left\{\begin{array}{l}
\frac{\Phi^{n+1}-\Phi^{n}}{\Delta t}=-\left(\frac{R^{n+1 / 2}}{\sqrt{E_{1, N}\left(\hat{\Phi}^{n+1 / 2}\right)}} N_{N}\left(\hat{\Phi}^{n+1 / 2}\right)+L_{N}\left(\frac{3}{4} \Phi^{n+1}+\frac{1}{4} \Phi^{n-1}\right)\right)+\tau_{\phi}^{n+1} \\
\frac{R^{n+1}-R^{n}}{\Delta t}=\frac{1}{2 \sqrt{E_{1, N}\left(\hat{\Phi}^{n+1 / 2}\right)}}\left\langle N_{N}\left(\hat{\Phi}^{n+1 / 2}\right), \frac{\Phi^{n+1}-\Phi^{n}}{\Delta t}\right\rangle+\tau_{r}^{n+1}
\end{array}\right.
$$

with

$$
\left\|\tau_{\phi}^{n+1 / 2}\right\|_{2},\left|\tau_{r}^{n+1 / 2}\right| \leq C\left(\Delta t^{2}+h^{m}\right)
$$

The derivation of (4.5) is accomplished with the help of the spectral approximation estimate and other related estimates; the details are left to interested readers.

The numerical error function is defined at a point-wise level:

$$
\begin{aligned}
& e^{k}:=\Phi^{k}-\phi^{k}, \quad \hat{e}^{k+1 / 2}:=\frac{3}{2} e^{k}-\frac{1}{2} e^{k-1}, \\
& \tilde{N}^{k+1 / 2}:=N_{N}\left(\hat{\Phi}^{k+1 / 2}\right)-N_{N}\left(\hat{\phi}^{k+1 / 2}\right), \quad \forall k \geq 0 .
\end{aligned}
$$

And also, the following scalar numerical errors are introduced

$$
\begin{aligned}
& \tilde{r}^{k}:=R^{k}-r^{k}, \quad \tilde{r}^{k+1 / 2}:=\frac{1}{2}\left(\tilde{r}^{k+1}+\tilde{r}^{k}\right), \\
& \tilde{E}_{1}^{k+1 / 2}:=E_{1, N}\left(\hat{\Phi}^{k+1 / 2}\right)-E_{1, N}\left(\hat{\phi}^{k+1 / 2}\right), \quad \forall k \geq 0 .
\end{aligned}
$$


In turn, subtracting the numerical scheme (2.24) from (4.5) gives

$$
\left\{\begin{aligned}
\frac{e^{n+1}-e^{n}}{\Delta t}=- & \left(\left(\frac{\tilde{r}^{n+1 / 2}}{\sqrt{E_{1, N}\left(\hat{\phi}^{n+1 / 2}\right)}}-B^{n+1 / 2} R^{n+1 / 2} \tilde{E}_{1}^{n+1 / 2}\right) N_{N}\left(\hat{\phi}^{n+1 / 2}\right)\right. \\
& \left.+\frac{R^{n+1 / 2}}{\sqrt{E_{1, N}\left(\hat{\Phi}^{n+1 / 2}\right)}} \tilde{N}^{n+1 / 2}+L_{N}\left(\frac{3}{4} e^{n+1}+\frac{1}{4} e^{n-1}\right)\right)+\tau_{\phi}^{n+1 / 2}, \\
\frac{\tilde{r}^{n+1}-\tilde{r}^{n}}{\Delta t}= & \frac{1}{2 \sqrt{E_{1, N}\left(\hat{\phi}^{n+1 / 2}\right)}}\left\langle N_{N}\left(\hat{\phi}^{n+1 / 2}\right), \frac{e^{n+1}-e^{n}}{\Delta t}\right\rangle \\
& +\frac{1}{2 \sqrt{E_{1, N}\left(\hat{\phi}^{n+1 / 2}\right)}}\left\langle\tilde{N}^{n+1 / 2}, \frac{\Phi^{n+1}-\Phi^{n}}{\Delta t}\right\rangle \\
& -\frac{1}{2} B^{n+1 / 2} \tilde{E}_{1}^{n+1 / 2}\left\langle N_{N}\left(\hat{\Phi}^{n+1 / 2}\right), \frac{\Phi^{n+1}-\Phi^{n}}{\Delta t}\right\rangle+\tau_{r}^{n+1 / 2}, \\
\text { with } B^{n+1 / 2}= & \frac{1}{\sqrt{E_{1, N}\left(\hat{\Phi}^{n+1 / 2}\right)} \sqrt{E_{1, N}\left(\hat{\phi}^{n+1 / 2}\right)}\left(\sqrt{E_{1, N}\left(\hat{\Phi}^{n+1 / 2}\right)}+\sqrt{E_{1, N}\left(\hat{\phi}^{n+1 / 2}\right)}\right)} .
\end{aligned}\right.
$$

\subsection{A few preliminary estimates}

The following estimates are needed in the later analysis.

Lemma 4.1. We have

$$
\begin{aligned}
& E_{1, N}\left(\hat{\phi}^{n+1 / 2}\right) \geq|\Omega|, \quad E_{1, N}\left(\hat{\Phi}^{n+1 / 2}\right) \geq|\Omega|, \quad 0 \leq B^{n+1 / 2} \leq \frac{1}{2}|\Omega|^{-\frac{3}{2}}, \\
& \left|\tilde{E}_{1}^{n+1 / 2}\right| \leq \tilde{C}_{2}\left\|\nabla_{N} \hat{e}^{n+1 / 2}\right\|_{2} \\
& \left\|N_{N}\left(\hat{\phi}^{n+1 / 2}\right)\right\| \leq \tilde{C}_{3} \\
& \left\|\tilde{N}^{n+1 / 2}\right\| \leq \tilde{C}_{4}\left\|\Delta_{N} \hat{e}^{n+1 / 2}\right\|_{2} \\
& \left\|\frac{\Phi^{n+1}-\Phi^{n}}{\Delta t}\right\|_{2} \leq C C^{*}
\end{aligned}
$$

in which

$$
\hat{e}^{n+1}:=\hat{\Phi}^{n+1}-\hat{\phi}^{n+1}=2 e^{n}-e^{n-1},
$$

and $\tilde{C}_{j}$ are independent of $\Delta t$ and $h, j=2,3,4$.

Proof. The lower bound for $E_{1, N}\left(\hat{\phi}^{n+1 / 2}\right)$ and $E_{1, N}\left(\hat{\Phi}^{n+1 / 2}\right)$ comes from their definition, and the estimate $0 \leq B^{n+1 / 2} \leq 2|\Omega|^{-\frac{3}{2}}$ is a direct result of its representation given by (4.8c). 
Moreover, a detailed expansion for $E_{1, N}\left(\hat{\phi}^{n+1 / 2}\right)$ and $E_{1, N}\left(\hat{\Phi}^{n+1 / 2}\right)$ implies that

$$
\begin{aligned}
\tilde{E}_{1}^{n+1 / 2}= & E_{1, N}\left(\hat{\Phi}^{n+1 / 2}\right)-E_{1, N}\left(\hat{\phi}^{n+1 / 2}\right) \\
= & \frac{1}{4}\left(\left\|\nabla_{N} \hat{\Phi}^{n+1 / 2}\right\|_{4}^{4}-\left\|\nabla_{N} \hat{\phi}^{n+1 / 2}\right\|_{4}^{4}\right)-\left(\left\|\nabla_{N} \hat{\Phi}^{n+1 / 2}\right\|_{2}^{2}-\left\|\nabla_{N} \hat{\phi}^{n+1 / 2}\right\|_{2}^{2}\right) \\
= & \frac{1}{4}\left\langle\left|\nabla_{N} \hat{\Phi}^{n+1 / 2}\right|^{2}+\left|\nabla_{N} \hat{\phi}^{n+1 / 2}\right|^{2}, \nabla_{N}\left(\hat{\Phi}^{n+1 / 2}+\hat{\phi}^{n+1 / 2}\right) \cdot \nabla_{N} \hat{e}^{n+1 / 2}\right\rangle \\
& \quad-\left\langle\nabla_{N}\left(\hat{\Phi}^{n+1 / 2}+\hat{\phi}^{n+1 / 2}\right), \nabla_{N} \hat{e}^{n+1 / 2}\right\rangle .
\end{aligned}
$$

For the first error expansion, an application of discrete Hölder inequality shows that

$$
\begin{aligned}
& \frac{1}{4}\left|\left\langle\left|\nabla_{N} \hat{\Phi}^{n+1 / 2}\right|^{2}+\left|\nabla_{N} \hat{\phi}^{n+1 / 2}\right|^{2}, \nabla_{N}\left(\hat{\Phi}^{n+1 / 2}+\hat{\phi}^{n+1 / 2}\right) \cdot \nabla_{N} \hat{e}^{n+1 / 2}\right\rangle\right| \\
\leq & \frac{1}{4}\left(\left\|\nabla_{N} \hat{\Phi}^{n+1 / 2}\right\|_{6}^{2}+\left\|\nabla_{N} \hat{\phi}^{n+1 / 2}\right\|_{6}^{2}\right) \cdot\left(\left\|\nabla_{N} \hat{\Phi}^{n+1 / 2}\right\|_{6}+\left\|\nabla_{N} \hat{\phi}^{n+1 / 2}\right\|_{6}\right) \cdot\left\|\nabla_{N} \hat{e}^{n+1 / 2}\right\|_{2} \\
\leq & \frac{1}{4}\left(4\left(C^{*}\right)^{2}+C \tilde{C}_{1}^{2}\right) \cdot\left(2 C^{*}+C \tilde{C}_{1}\right) \cdot\left\|\nabla_{N} \hat{e}^{n+1 / 2}\right\|_{2} \\
\leq & C\left(\left(C^{*}\right)^{3}+\tilde{C}_{1}^{3}\right)\left\|\nabla_{N} \hat{e}^{n+1 / 2}\right\|_{2}
\end{aligned}
$$

in which the regularity assumption (4.2) for the exact solution and the discrete $W^{1,6}$ bound (2.47) for the numerical solution have been applied. The second error expansion term in (4.10) could be controled in an even simpler way:

$$
\begin{aligned}
& \left|\left\langle\mid \nabla_{N}\left(\hat{\Phi}^{n+1 / 2}+\hat{\phi}^{n+1 / 2}\right), \nabla_{N} \hat{e}^{n+1 / 2}\right\rangle\right| \\
\leq & \left(\left\|\nabla_{N} \hat{\Phi}^{n+1 / 2}\right\|_{2}+\left\|\nabla_{N} \hat{\phi}^{n+1 / 2}\right\|_{2}\right) \cdot\left\|\nabla_{N} \hat{e}^{n+1 / 2}\right\|_{2} \\
\leq & \left(2 C^{*}+C \tilde{C}_{1}\right)\left\|\nabla_{N} \hat{e}^{n+1 / 2}\right\|_{2},
\end{aligned}
$$

with (4.2), (2.47), applied again. This comletes the proof of inequality (4.9b), by setting

$$
\tilde{C}_{2}:=C\left(\left(C^{*}\right)^{3}+\tilde{C}_{1}^{3}+C^{*}+\tilde{C}_{1}\right) .
$$

To obtain a discrete $\ell^{2}$ estimate for $N_{N}\left(\hat{\phi}^{n+1 / 2}\right)$, we recall the grid function $\hat{q}^{n+1 / 2}$ introduced in (3.10), so that the following identity is valid:

$$
\left\|\nabla_{N} \cdot\left(\left|\nabla_{N} \hat{\phi}^{n+1 / 2}\right|^{2} \nabla_{N} \hat{\phi}^{n+1 / 2}\right)\right\|_{2}=\left\|\nabla\left(\nabla \cdot \hat{q}_{S}^{n+1 / 2}\right)\right\|_{L^{2}}
$$

in which $\hat{q}_{S}^{n+1 / 2}$ is the spectral interpolation of $\hat{q}^{n+1 / 2}$. Because of the fact $\hat{q}_{S}^{n+1 / 2}=$ $R_{N}\left(\varphi_{\hat{q}^{n+1 / 2}}\right)$, as indicated by the point-wise interpolation given by (3.12), we make use of the aliasing error control inequality in Lemma 2.1 and get

$$
\left\|\nabla \cdot \hat{q}_{S}^{n+1 / 2}\right\|_{L^{2}} \leq\left\|\hat{q}_{S}^{n+1 / 2}\right\|_{H^{1}}=\left\|R_{N}\left(\varphi_{\hat{q}^{n+1 / 2}}\right)\right\|_{H^{1}} \leq 3\left\|\varphi_{\hat{q}^{n+1 / 2}}\right\|_{H^{1}},
$$


an inequality similar to (3.13). Moreover, a detailed expansion and repeated applications of Hölder inequality lead to

$$
\begin{aligned}
\left\|\varphi_{\hat{q}^{n+1 / 2}}\right\|_{H^{1}} & \leq C\left(\left\|\varphi_{\hat{q}^{n+1 / 2}}\right\|+\left\|\nabla \varphi_{\hat{q}^{n+1 / 2}}\right\|\right) \\
& \leq C\left(\left\|\left|\nabla \hat{\phi}_{S}^{n+1 / 2}\right|^{2} \nabla \hat{\phi}_{S}^{n+1 / 2}\right\|+\left\|\nabla\left(\left|\nabla \hat{\phi}_{S}^{n+1 / 2}\right|^{2} \nabla \hat{\phi}_{S}^{n+1 / 2}\right)\right\|\right) \\
& \leq C\left\|\nabla \hat{\phi}_{S}^{n+1}\right\|_{L^{\infty}}^{2} \cdot\left\|\nabla \hat{\phi}_{S}^{n+1}\right\|_{H^{1}} \leq C\left\|\hat{\phi}_{S}^{n+1}\right\|_{H^{3}}^{3} \leq C\left(Q^{(3)}\right)^{3},
\end{aligned}
$$

in which the uniform in time $H^{3}$ estimate (3.1) (for the numerical solution) has been applied in the last step. Going back (4.14) and (4.13), we arrive at

$$
\left\|\nabla_{N} \cdot\left(\left|\nabla_{N} \hat{\phi}^{n+1 / 2}\right|^{2} \nabla_{N} \hat{\phi}^{n+1 / 2}\right)\right\|_{2} \leq C\left(Q^{(3)}\right)^{3} .
$$

The other expansion term in $N_{N}\left(\hat{\phi}^{n+1}\right)$ could be bounded in a more standard way:

$$
\left\|\Delta_{N} \hat{\phi}^{n+1 / 2}\right\|_{2} \leq\left\|\hat{\phi}_{S}^{n+1 / 2}\right\|_{H^{3}} \leq 2 Q^{(3)} .
$$

Therefore, a combination of (4.16) and (4.17) gives the inequality (4.9c), by taking $\tilde{C}_{3}=$ $C\left(Q^{(3)}\right)^{3}+2 Q^{(3)}$.

Inequality (4.9d) could be derived in a similar manner. Making a comparison between $N_{N}\left(\hat{\Phi}^{n+1 / 2}\right)$ and $N_{N}\left(\hat{\phi}^{n+1 / 2}\right)$, we observe that $\tilde{N}^{n+1 / 2}$ turns out to be the point-wise interpolation of the following continuous function

$$
\begin{aligned}
& \tilde{N}_{S}^{n+1 / 2}=-\nabla \cdot\left(R_{N}\left(\varphi_{\tilde{N}^{n+1 / 2}}\right)+\Delta \hat{e}_{S}^{n+1 / 2},\right. \\
& \varphi_{\tilde{N}^{n+1 / 2}}:=\left|\nabla \hat{\Phi}_{S}^{n+1 / 2}\right|^{2} \nabla \hat{\Phi}_{S}^{n+1 / 2}-\left|\nabla \hat{\phi}_{S}^{n+1 / 2}\right|^{2} \nabla \hat{\phi}_{S}^{n+1 / 2},
\end{aligned}
$$

with

$$
\hat{\Phi}_{S}^{n+1 / 2}=\frac{3}{2} \Phi_{S}^{n}-\frac{1}{2} P h i_{S}^{n-1}, \quad \hat{e}_{S}^{n+1 / 2}=\frac{3}{2} e_{S}^{n}-\frac{1}{2} e_{S}^{n-1} .
$$

A similar expansion is available for $\varphi_{\tilde{N}^{n+1}}$ :

$$
\varphi_{\tilde{N}^{n+1 / 2}}=\left|\nabla \hat{\Phi}_{S}^{n+1 / 2}\right|^{2} \nabla \hat{e}_{S}^{n+1 / 2}+\left(\nabla\left(\hat{\Phi}_{S}^{n+1 / 2}+\hat{\phi}_{S}^{n+1 / 2}\right) \cdot \nabla \hat{e}_{S}^{n+1 / 2}\right) \nabla \hat{\phi}_{S}^{n+1 / 2} .
$$

Again, repeated applications of Hölder inequality gives the following estimate

$$
\begin{aligned}
& \left\|\varphi_{\tilde{N}^{n+1 / 2}}\right\|_{H^{1}} \\
\leq & C\left(\left\|\varphi_{\tilde{N}^{n+1 / 2}}\right\|+\left\|\nabla \varphi_{\tilde{N}^{n+1 / 2}}\right\|\right) \\
\leq & C\left(\left\|\left|\nabla \hat{\Phi}_{S}^{n+1 / 2}\right|^{2} \nabla \hat{e}_{S}^{n+1 / 2}\right\|+\left\|\left(\nabla\left(\hat{\Phi}_{S}^{n+1 / 2}+\hat{\phi}_{S}^{n+1 / 2}\right) \cdot \nabla \hat{e}_{S}^{n+1 / 2}\right) \nabla \hat{\phi}_{S}^{n+1 / 2}\right\|\right. \\
& \left.\quad+\left\|\nabla\left(\left|\nabla \hat{\Phi}_{S}^{n+1 / 2}\right|^{2} \nabla \hat{e}_{S}^{n+1 / 2}\right)\right\|+\left\|\nabla\left(\left(\nabla\left(\hat{\Phi}_{S}^{n+1 / 2}+\hat{\phi}_{S}^{n+1 / 2}\right) \cdot \nabla \hat{e}_{S}^{n+1 / 2}\right) \nabla \hat{\phi}_{S}^{n+1 / 2}\right)\right\|\right) \\
\leq & C\left(\left\|\nabla \hat{\Phi}_{S}^{n+1 / 2}\right\|_{H^{2}}+\left\|\nabla \hat{\phi}_{S}^{n+1 / 2}\right\|_{H^{2}}\right)^{2} \cdot\left\|\nabla \hat{e}_{S}^{n+1 / 2}\right\|_{H^{1}} \\
\leq & C\left(\left(C^{*}\right)^{2}+\left(Q^{(3)}\right)^{2}\right)\left\|\nabla \hat{e}_{S}^{n+1 / 2}\right\|_{H^{1}},
\end{aligned}
$$


with the uniform in time $H^{3}$ estimate (3.1) and the regularity assumption (4.2) recalled. Since $\varphi_{\tilde{N}^{n+1}} \in \mathcal{P}_{3 K}$, we go back (4.18) and arrive at

$$
\begin{aligned}
\left\|\tilde{N}^{n+1 / 2}\right\|_{2} & =\left\|\tilde{N}_{S}^{n+1 / 2}\right\|=\left\|-\nabla \cdot\left(R_{N}\left(\varphi_{\tilde{N}^{n+1 / 2}}\right)\right)+\Delta \hat{e}_{S}^{n+1 / 2}\right\| \\
& \leq 3\left\|\varphi_{\tilde{N}^{n+1 / 2}}\right\|_{H^{1}}+\left\|\Delta \hat{e}_{S}^{n+1 / 2}\right\| \\
& \leq C\left(\left(C^{*}\right)^{2}+\left(Q^{(3)}\right)^{2}\right)\left\|\nabla \hat{e}_{S}^{n+1 / 2}\right\|_{H^{1}}+\left\|\nabla \hat{e}_{S}^{n+1 / 2}\right\|_{H^{1}} \\
& \leq C\left(\left(C^{*}\right)^{2}+\left(Q^{(3)}\right)^{2}+1\right)\left\|\nabla \hat{e}_{S}^{n+1 / 2}\right\|_{H^{1}} \\
& \leq C\left(\left(C^{*}\right)^{2}+\left(Q^{(3)}\right)^{2}+1\right)\left\|\Delta \hat{e}_{S}^{n+1 / 2}\right\| \\
& \leq C\left(\left(C^{*}\right)^{2}+\left(Q^{(3)}\right)^{2}+1\right)\left\|\Delta_{N} \hat{e}^{n+1}\right\|_{2},
\end{aligned}
$$

in which the elliptic regularity,

$$
\left\|\nabla \hat{e}_{S}^{n+1 / 2}\right\|_{H^{1}} \leq C\left\|\Delta \hat{e}_{S}^{n+1 / 2}\right\|
$$

has been applied in the fourth step, due to the fact that

$$
\int_{\Omega} \nabla \hat{e}_{S}^{n+1 / 2} d \mathbf{x}=0
$$

and the last step comes from the fact that $\hat{e}_{S}^{n+1 / 2}$ is the spectral interpolation function of $\hat{e}^{n+1 / 2}$. This completes the proof of inequality (4.9d), by setting

$$
\tilde{C}_{4}=C\left(\left(C^{*}\right)^{2}+\left(Q^{(3)}\right)^{2}+1\right) .
$$

The last inequality (4.9e) is a direct consequence of the following estimates

$$
\left\|\frac{\Phi^{n+1}-\Phi^{n}}{\Delta t}\right\|_{\infty} \leq C^{*} \quad \text { by }(4.2),
$$

combined with the fact that $\|\cdot\|_{\infty}$ is a norm stronger than $\|\cdot\|_{2}$.

\subsection{Proof of the convergence theorem}

Now we proceed into the proof of Theorem 4.1.

Proof. Taking a discrete inner product of (4.8a) with $\frac{e^{n+1}-e^{n}}{\Delta t}$, with a repeated application of summation by parts, we get

$$
\begin{aligned}
& \frac{1}{\Delta t}\left\langle e^{n+1}-e^{n}, L_{N}\left(\frac{3}{4} e^{n+1}+\frac{1}{4} e^{n-1}\right)\right\rangle+\left\|\frac{e^{n+1}-e^{n}}{\Delta t}\right\|_{2}^{2} \\
& =-\left\langle\left(\mathcal{N} \mathcal{L} \mathcal{E}_{1}+\mathcal{N} \mathcal{L} \mathcal{E}_{2}+\mathcal{N} \mathcal{L} \mathcal{E}_{3}-\tau_{\phi}^{n+1 / 2}\right), \frac{e^{n+1}-e^{n}}{\Delta t}\right\rangle,
\end{aligned}
$$




$$
\begin{aligned}
& \mathcal{N} \mathcal{L} \mathcal{E}_{1}=\frac{\tilde{r}^{n+1 / 2}}{\sqrt{E_{1, N}\left(\hat{\phi}^{n+1 / 2}\right)}} N_{N}\left(\hat{\phi}^{n+1 / 2}\right), \\
& \mathcal{N} \mathcal{L} \mathcal{E}_{2}=-B^{n+1 / 2} R^{n+1 / 2} \tilde{E}_{1}^{n+1 / 2} N_{N}\left(\hat{\phi}^{n+1 / 2}\right), \\
& \mathcal{N} \mathcal{L} \mathcal{E}_{3}=\frac{R^{n+1 / 2}}{\sqrt{E_{1, N}\left(\hat{\Phi}^{n+1 / 2}\right)}} \tilde{N}^{n+1 / 2} .
\end{aligned}
$$

The temporal stencil term could be analyzed in the same manner as (2.39a):

$$
\begin{aligned}
& \left\langle L_{N}\left(\frac{3}{4} e^{n+1}+\frac{1}{4} e^{n-1}\right), \phi^{n+1}-\phi^{n}\right\rangle \\
= & \frac{1}{2}\left(\left\|L_{N}^{\frac{1}{2}} e^{n+1}\right\|_{2}^{2}-\left\|L_{N}^{\frac{1}{2}} e^{n}\right\|_{2}^{2}\right)+\frac{1}{8}\left(\left\|L_{N}^{\frac{1}{2}}\left(e^{n+1}-e^{n}\right)\right\|_{2}^{2}-\left\|L_{N}^{\frac{1}{2}}\left(e^{n}-e^{n-1}\right)\right\|_{2}^{2}\right) \\
& +\frac{1}{8}\left(\| L_{N}^{\frac{1}{2}}\left(e^{n+1}-2 e^{n}+e^{n-1} \|_{2}^{2}\right) .\right.
\end{aligned}
$$

A bound for the truncation error inner product term is standard:

$$
\left\langle\tau_{\phi}^{n+1 / 2}, \frac{e^{n+1}-e^{n}}{\Delta t}\right\rangle \leq\left\|\tau_{\phi}^{n+1}\right\|_{2} \cdot\left\|\frac{e^{n+1}-e^{n}}{\Delta t}\right\|_{2} \leq 2\left\|\tau_{\phi}^{n+1 / 2}\right\|_{2}^{2}+\frac{1}{8}\left\|\frac{e^{n+1}-e^{n}}{\Delta t}\right\|_{2}^{2} .
$$

The first nonlinear inner product term could be rewritten as follows:

$$
-\left\langle\mathcal{N} \mathcal{L} \mathcal{E}_{1}, \frac{e^{n+1}-e^{n}}{\Delta t}\right\rangle=-\left\langle\frac{\tilde{r}^{n+1}}{\sqrt{E_{1, N}\left(\hat{\phi}^{n+1 / 2}\right)}} N_{N}\left(\hat{\phi}^{n+1 / 2}\right), \frac{e^{n+1}-e^{n}}{\Delta t}\right\rangle .
$$

For the second and third nonlinear inner product terms, we begin with the following estimates:

$$
\begin{aligned}
& \left\|\mathcal{N} \mathcal{L} \mathcal{E}_{2}\right\|_{2}=\left\|B^{n+1 / 2} R^{n+1 / 2} \tilde{E}_{1}^{n+1 / 2} N_{N}\left(\hat{\phi}^{n+1 / 2}\right)\right\|_{2} \\
& \leq\left|B^{n+1 / 2}\right| \cdot\left|R^{n+1 / 2}\right| \cdot\left|\tilde{E}_{1}^{n+1 / 2}\right| \cdot\left\|N_{N}\left(\hat{\phi}^{n+1}\right)\right\|_{2} \\
& \leq \frac{1}{2}|\Omega|^{-\frac{3}{2}} \cdot\left(\tilde{C}_{0}+1\right)^{\frac{1}{2}} \cdot \tilde{C}_{2}\left\|\nabla_{N} \hat{e}^{n+1 / 2}\right\|_{2} \cdot \tilde{C}_{3} \\
& =\tilde{C}_{5}\left\|\nabla_{N} \hat{e}^{n+1 / 2}\right\|_{2} \quad \text { with } \tilde{C}_{5}=\frac{1}{2} \tilde{C}_{2} \tilde{C}_{3}\left(\tilde{C}_{0}+1\right)^{\frac{1}{2}}|\Omega|^{-\frac{3}{2}}, \\
& \left\|\mathcal{N} \mathcal{L} \mathcal{E}_{3}\right\|_{2}=\left\|R^{n+1 / 2}\left(E_{1, N}\left(\hat{\Phi}^{n+1 / 2}\right)\right)^{-\frac{1}{2}} \tilde{N}^{n+1 / 2}\right\|_{2} \\
& \leq\left|R^{n+1 / 2}\right| \cdot|\Omega|^{-\frac{1}{2}} \cdot\left\|\tilde{N}^{n+1}\right\|_{2} \\
& \leq\left(\tilde{C}_{0}+1\right)^{\frac{1}{2}}|\Omega|^{-\frac{1}{2}} \cdot \tilde{C}_{4}\left\|\Delta_{N} \hat{e}^{n+1 / 2}\right\|_{2} \\
& =\tilde{C}_{6}\left\|\Delta_{N} \hat{e}^{n+1 / 2}\right\|_{2} \quad \text { with } \tilde{C}_{6}=\tilde{C}_{4}\left(\tilde{C}_{0}+1\right)^{\frac{1}{2}}|\Omega|^{-\frac{1}{2}},
\end{aligned}
$$


in which the preliminary estimates (4.9a)-(4.9d) in Lemma 4.1 have been extensively applied in the derivation. We also notice that the inequality $\left|R^{n+1}\right|,\left|R^{n}\right| \leq\left(\tilde{C}_{0}+1\right)^{\frac{1}{2}}$ comes from the fact that

$$
E(\Phi(t)) \leq E\left(\Phi^{0}\right)=\tilde{C}_{0}+h^{m},
$$

the pseudo-spectral approximation order, combined with the inequality

$$
E_{1, N}\left(\Phi^{k}\right) \leq E_{N}\left(\Phi^{k}\right)
$$

And also, the following estimate for $\left\|\mathcal{N} \mathcal{L} \mathcal{E}_{1}\right\|_{2}$ is derived below, which will be needed in the later analysis:

$$
\begin{aligned}
\left\|\mathcal{N} \mathcal{L} \mathcal{E}_{1}\right\|_{2} & =\left\|\tilde{r}^{n+1 / 2}\left(E_{1, N}\left(\hat{\phi}^{n+1 / 2}\right)\right)^{-\frac{1}{2}} N_{N}\left(\hat{\phi}^{n+1 / 2}\right)\right\|_{2} \\
& \leq\left|\tilde{r}^{n+1 / 2}\right| \cdot|\Omega|^{-\frac{1}{2}} \cdot\left\|N_{N}\left(\hat{\phi}^{n+1 / 2}\right)\right\|_{2} \\
& \leq|\Omega|^{-\frac{1}{2}} \cdot \tilde{C}_{3} \cdot \tilde{r}^{n+1 / 2}=\tilde{C}_{7} \tilde{r}^{n+1 / 2} \quad \text { with } \tilde{C}_{7}=\tilde{C}_{3}|\Omega|^{-\frac{1}{2}} .
\end{aligned}
$$

As a consequence of $(4.27 \mathrm{a}),(4.27 \mathrm{~b})$, the following inequalities are available:

$$
\begin{aligned}
& -\left\langle\mathcal{N} \mathcal{L} \mathcal{E}_{2}+\mathcal{N} \mathcal{L} \mathcal{E}_{3}, \frac{e^{n+1}-e^{n}}{\Delta t}\right\rangle \\
\leq & \left(\left\|\mathcal{N} \mathcal{L} \mathcal{E}_{2}\right\|_{2}+\left\|\mathcal{N} \mathcal{L} \mathcal{E}_{3}\right\|_{2}\right) \cdot\left\|\frac{e^{n+1}-e^{n}}{\Delta t}\right\|_{2} \\
\leq & 2\left(\left\|\mathcal{N} \mathcal{L} \mathcal{E}_{2}\right\|_{2}^{2}+\left\|\mathcal{N} \mathcal{L} \mathcal{E}_{3}\right\|_{2}^{2}\right)+\frac{1}{4}\left\|\frac{e^{n+1}-e^{n}}{\Delta t}\right\|_{2}^{2} \\
\leq & 2\left(\tilde{C}_{5}^{2}\left\|\nabla_{N} \hat{e}^{n+1 / 2}\right\|_{2}^{2}+\tilde{C}_{6}^{2}\left\|\Delta_{N} \hat{e}^{n+1 / 2}\right\|_{2}^{2}\right)+\frac{1}{4}\left\|\frac{e^{n+1}-e^{n}}{\Delta t}\right\|_{2}^{2} \\
\leq & \tilde{C}_{8}\left\|\Delta_{N} \hat{e}^{n+1 / 2}\right\|_{2}^{2}+\frac{1}{4}\left\|\frac{e^{n+1}-e^{n}}{\Delta t}\right\|_{2}^{2} \quad \tilde{C}_{8}=2\left(\tilde{C}_{5}^{2} C_{3}^{2}+\tilde{C}_{6}^{2}\right),
\end{aligned}
$$

in which $C_{3}$ corresponds to the elliptic regularity,

$$
\left\|\nabla_{N} f\right\|_{2} \leq C_{3}\left\|\Delta_{N} f\right\|_{2}
$$

an inequality similar to (3.26). Therefore, a substitution of (4.24)-(4.26) and (4.29) into (4.23a) yields

$$
\begin{aligned}
& \frac{1}{2 \Delta t}\left(\left\|L_{N}^{\frac{1}{2}} e^{n+1}\right\|_{2}^{2}-\left\|L_{N}^{\frac{1}{2}} e^{n}\right\|_{2}^{2}\right)+\frac{1}{8}\left(\left\|L_{N}^{\frac{1}{2}}\left(e^{n+1}-e^{n}\right)\right\|_{2}^{2}-\left\|L_{N}^{\frac{1}{2}}\left(e^{n}-e^{n-1}\right)\right\|_{2}^{2}\right) \\
& +\frac{5}{8}\left\|\frac{e^{n+1}-e^{n}}{\Delta t}\right\|_{2}^{2} \\
\leq & -\left\langle\frac{\tilde{r}^{n+1 / 2}}{\sqrt{E_{1, N}\left(\hat{\phi}^{n+1 / 2}\right)}} N_{N}\left(\hat{\phi}^{n+1 / 2}\right), \frac{e^{n+1}-e^{n}}{\Delta t}\right\rangle+\tilde{C}_{8}\left\|\Delta_{N} \hat{e}^{n+1 / 2}\right\|_{2}^{2}+2\left\|\tau_{\phi}^{n+1}\right\|_{2}^{2} .
\end{aligned}
$$


On the other hand, the original error evolutionary equation (4.8a) gives

$$
\frac{e^{n+1}-e^{n}}{\Delta t}=-\left(L_{N}\left(\frac{3}{4} e^{n+1}+\frac{1}{4} e^{n-1}\right)+\mathcal{N} \mathcal{L} \mathcal{E}_{1}+\mathcal{N} \mathcal{L} \mathcal{E}_{2}+\mathcal{N} \mathcal{L} \mathcal{E}_{3}-\tau_{\phi}^{n+1}\right)
$$

In turn, an application of quadratic inequality implies that

$$
\begin{aligned}
& \left\|\frac{e^{n+1}-e^{n}}{\Delta t}\right\|_{2}^{2} \\
\geq & \frac{1}{2}\left\|L_{N}\left(\frac{3}{4} e^{n+1}+\frac{1}{4} e^{n-1}\right)\right\|_{2}^{2}-2\left\|\mathcal{N} \mathcal{L} \mathcal{E}_{1}+\mathcal{N} \mathcal{L} \mathcal{E}_{2}+\mathcal{N} \mathcal{L} \mathcal{E}_{3}-\tau_{\phi}^{n+1}\right\|_{2}^{2} \\
\geq & \frac{1}{2}\left\|L_{N}\left(\frac{3}{4} e^{n+1}+\frac{1}{4} e^{n-1}\right)\right\|_{2}^{2}-4\left(\left\|\mathcal{N} \mathcal{L} \mathcal{E}_{1}+\mathcal{N} \mathcal{L} \mathcal{E}_{2}+\mathcal{N} \mathcal{L} \mathcal{E}_{3}\right\|_{2}^{2}+\left\|\tau_{\phi}^{n+1}\right\|_{2}^{2}\right) \\
\geq & \frac{1}{2}\left\|L_{N}\left(\frac{3}{4} e^{n+1}+\frac{1}{4} e^{n-1}\right)\right\|_{2}^{2}-12\left(\left\|\mathcal{N} \mathcal{L} \mathcal{E}_{1}\right\|_{2}^{2}+\left\|\mathcal{N} \mathcal{L} \mathcal{E}_{2}\right\|_{2}^{2}+\left\|\mathcal{N} \mathcal{L} \mathcal{E}_{3}\right\|_{2}^{2}\right)-4\left\|\tau_{\phi}^{n+1}\right\|_{2}^{2} \\
\geq & \frac{1}{2}\left\|L_{N}\left(\frac{3}{4} e^{n+1}+\frac{1}{4} e^{n-1}\right)\right\|_{2}^{2}-12\left(\tilde{C}_{7}^{2}\left(\tilde{r}^{n+1 / 2}\right)^{2}\right. \\
& \left.\quad+\left(\tilde{C}_{5}^{2} C_{3}^{2}+\tilde{C}_{6}^{2}\right)\left\|\Delta_{N} \hat{e}^{n+1 / 2}\right\|_{2}^{2}\right)-4\left\|\tau_{\phi}^{n+1}\right\|_{2}^{2}
\end{aligned}
$$

with the estimates (4.27a)-(4.28) recalled. Going back (4.30), we arrive at

$$
\begin{gathered}
\frac{1}{2 \Delta t}\left(\left\|L_{N}^{\frac{1}{2}} e^{n+1}\right\|_{2}^{2}-\left\|L_{N}^{\frac{1}{2}} e^{n}\right\|_{2}^{2}\right)+\frac{1}{8}\left(\left\|L_{N}^{\frac{1}{2}}\left(e^{n+1}-e^{n}\right)\right\|_{2}^{2}-\left\|L_{N}^{\frac{1}{2}}\left(e^{n}-e^{n-1}\right)\right\|_{2}^{2}\right) \\
\quad+\frac{5}{16}\left\|L_{N}\left(\frac{3}{4} e^{n+1}+\frac{1}{4} e^{n-1}\right)\right\|_{2}^{2}+\left\langle\frac{\tilde{r}^{n+1 / 2}}{\sqrt{E_{1, N}\left(\hat{\phi}^{n+1 / 2}\right)}} N_{N}\left(\hat{\phi}^{n+1 / 2}\right), \frac{e^{n+1}-e^{n}}{\Delta t}\right\rangle \\
\leq 12 \tilde{C}_{7}^{2}\left(\tilde{r}^{n+1 / 2}\right)^{2}+7 \tilde{C}_{8}\left\|\Delta_{N} \hat{e}^{n+1 / 2}\right\|_{2}^{2}+6\left\|\tau_{\phi}^{n+1 / 2}\right\|_{2}^{2} .
\end{gathered}
$$

Taking a discrete inner product of $(4.8 \mathrm{~b})$ with $2 \tilde{r}^{n+1 / 2}=\tilde{r}^{n+1}+\tilde{r}^{n}$ gives

$$
\begin{aligned}
& \frac{1}{\Delta t}\left(\left|\tilde{r}^{n+1}\right|^{2}-\left|\tilde{r}^{n}\right|^{2}\right) \\
= & \frac{\tilde{r}^{n+1 / 2}}{\sqrt{E_{1, N}\left(\hat{\phi}^{n+1 / 2}\right)}}\left\langle N_{N}\left(\hat{\phi}^{n+1 / 2}\right), \frac{e^{n+1}-e^{n}}{\Delta t}\right\rangle+\frac{\tilde{r}^{n+1 / 2}}{\sqrt{E_{1, N}\left(\hat{\phi}^{n+1 / 2}\right)}}\left\langle\tilde{N}^{n+1 / 2}, \frac{\Phi^{n+1}-\Phi^{n}}{\Delta t}\right\rangle \\
& -B^{n+1 / 2} \tilde{E}_{1}^{n+1 / 2} \tilde{r}^{n+1 / 2}\left\langle N_{N}\left(\hat{\Phi}^{n+1 / 2}\right), \frac{\Phi^{n+1}-\Phi^{n}}{\Delta t}\right\rangle+2 \tau_{r}^{n+1 / 2} \cdot \tilde{r}^{n+1 / 2} .
\end{aligned}
$$

The inner product associated with the truncation error could be controlled via Cauchy inequality:

$$
2 \tau_{r}^{n+1 / 2} \cdot \tilde{r}^{n+1 / 2} \leq\left|\tau_{r}^{n+1 / 2}\right|^{2}+\left|\tilde{r}^{n+1 / 2}\right|^{2} .
$$


The first nonlinear inner product on the right hand side is kept. The second and third nonlinear inner product terms could be analyzed as follows

$$
\begin{aligned}
& \frac{\tilde{r}^{n+1 / 2}}{\sqrt{E_{1, N}\left(\hat{\phi}^{n+1 / 2}\right)}}\left\langle\tilde{N}^{n+1 / 2}, \frac{\Phi^{n+1}-\Phi^{n}}{\Delta t}\right\rangle \leq\left|\tilde{r}^{n+1 / 2}\right| \cdot|\Omega|^{-\frac{1}{2}} \cdot\left\|\tilde{N}^{n+1}\right\|_{2} \cdot\left\|\frac{\Phi^{n+1}-\Phi^{n}}{\Delta t}\right\|_{2} \\
& \leq\left|\tilde{r}^{n+1 / 2}\right| \cdot|\Omega|^{-\frac{1}{2}} \cdot \tilde{C}_{4}\left\|\Delta_{N} \hat{e}^{n+1 / 2}\right\|_{2} \cdot C C^{*} \leq \tilde{C}_{9}\left|\tilde{r}^{n+1 / 2}\right| \cdot\left\|\Delta_{N} \hat{e}^{n+1 / 2}\right\|_{2} \\
& \leq \frac{\tilde{C}_{9}}{2}\left(\left|\tilde{r}^{n+1 / 2}\right|^{2}+\left\|\Delta_{N} \hat{e}^{n+1 / 2}\right\|_{2}^{2}\right), \quad \tilde{C}_{9}=C \tilde{C}_{4} C^{*}|\Omega|^{-\frac{1}{2}} \\
& -B^{n+1 / 2} \tilde{E}_{1}^{n+1 / 2} \tilde{r}^{n+1 / 2}\left\langle N_{N}\left(\hat{\Phi}^{n+1 / 2}\right), \frac{\Phi^{n+1}-\Phi^{n}}{\Delta t}\right\rangle \\
& \leq\left|B^{n+1 / 2}\right| \cdot\left|\tilde{E}_{1}^{n+1 / 2}\right| \cdot\left|\tilde{r}^{n+1 / 2}\right| \cdot\left\|N_{N}\left(\hat{\Phi}^{n+1 / 2}\right)\right\|_{2} \cdot\left\|\frac{\Phi^{n+1}-\Phi^{n}}{\Delta t}\right\|_{2} \\
& \leq \frac{1}{2}|\Omega|^{-\frac{3}{2}} \cdot \tilde{C}_{2}\left\|\nabla_{N} \hat{e}^{n+1 / 2}\right\|_{2} \cdot\left|\tilde{r}^{n+1 / 2}\right| \cdot \tilde{C}_{3} \cdot C C^{*} \leq \tilde{C}_{10}\left|\tilde{r}^{n+1 / 2}\right| \cdot\left\|\Delta_{N} \hat{e}^{n+1 / 2}\right\|_{2} \\
& \leq \frac{\tilde{C}_{10}}{2}\left(\left|\tilde{r}^{n+1 / 2}\right|^{2}+\left\|\Delta_{N} \hat{e}^{n+1 / 2}\right\|_{2}^{2}\right), \quad \tilde{C}_{10}=C \tilde{C}_{2} \tilde{C}_{3} C_{3} C^{*}|\Omega|^{-\frac{3}{2}}
\end{aligned}
$$

with repeated application of the preliminary estimates (4.9a)-(4.9e) in Lemma 4.1. Subsequently, a substitution of (4.35)-(4.36b) into (4.34) yields

$$
\begin{aligned}
& \frac{1}{\Delta t}\left(\left|\tilde{r}^{n+1}\right|^{2}-\left|\tilde{r}^{n}\right|^{2}\right) \\
\leq & \frac{\tilde{r}^{n+1 / 2}}{\sqrt{E_{1, N}\left(\hat{\phi}^{n+1 / 2}\right)}}\left\langle N_{N}\left(\hat{\phi}^{n+1 / 2}\right), \frac{e^{n+1}-e^{n}}{\Delta t}\right\rangle \\
& \quad+\frac{\tilde{C}_{9}+\tilde{C}_{10}}{2}\left(\left|\tilde{r}^{n+1 / 2}\right|^{2}+\left\|\Delta_{N} \hat{e}^{n+1 / 2}\right\|_{2}^{2}\right)+\left|\tilde{r}^{n+1 / 2}\right|^{2}+\left|\tau_{r}^{n+1 / 2}\right|^{2} .
\end{aligned}
$$

Finally, a combination of (4.33) and (4.37) results in

$$
\begin{aligned}
& \frac{1}{2 \Delta t}\left(\left\|L_{N}^{\frac{1}{2}} e^{n+1}\right\|_{2}^{2}-\left\|L_{N}^{\frac{1}{2}} e^{n}\right\|_{2}^{2}\right)+\frac{1}{8}\left(\left\|L_{N}^{\frac{1}{2}}\left(e^{n+1}-e^{n}\right)\right\|_{2}^{2}-\left\|L_{N}^{\frac{1}{2}}\left(e^{n}-e^{n-1}\right)\right\|_{2}^{2}\right) \\
& +\frac{5}{16}\left\|L_{N}\left(\frac{3}{4} e^{n+1}+\frac{1}{4} e^{n-1}\right)\right\|_{2}^{2}+\frac{1}{\Delta t}\left(\left|\tilde{r}^{n+1}\right|^{2}-\left|\tilde{r}^{n}\right|^{2}\right) \\
\leq & \tilde{C}_{11}\left|\tilde{r}^{n+1 / 2}\right|^{2}+\tilde{C}_{12}\left\|\Delta_{N} \hat{e}^{n+1 / 2}\right\|_{2}^{2}+6\left\|\tau_{\phi}^{n+1 / 2}\right\|_{2}^{2}+\left|\tau_{r}^{n+1 / 2}\right|^{2}
\end{aligned}
$$

with

$$
\tilde{C}_{11}=12\left(\tilde{C}_{7}^{2}+\tilde{C}_{9}^{2}+\tilde{C}_{10}^{2}\right)+1, \quad \tilde{C}_{12}=7 \tilde{C}_{8}+\frac{\tilde{C}_{9}+\tilde{C}_{10}}{2} .
$$

In particular, we notice that the first nonlinear error inner product terms have been cancelled; this subtle fact has played a crucial role in the analysis. In addition, the following 
inequality is observed:

$$
\begin{aligned}
\left\|\Delta_{N} \hat{e}^{n+1 / 2}\right\|_{2}^{2} & =\left\|\Delta_{N}\left(\frac{3}{2} e^{n}-\frac{1}{2} e^{n-1}\right)\right\|_{2}^{2} \leq 3\left\|\Delta_{N} e^{n}\right\|_{2}^{2}+\left\|\Delta_{N} e^{n-1}\right\|_{2}^{2} \\
& \leq \varepsilon^{-2}\left(3\left\|L_{N}^{\frac{1}{2}} e^{n}\right\|_{2}^{2}+\left\|L_{N}^{\frac{1}{2}} e^{n-1}\right\|_{2}^{2}\right),
\end{aligned}
$$

in which we have used the fact that

$$
\left\|\Delta_{N} f\right\|_{2}=\varepsilon^{-1}\left\|L_{N}^{\frac{1}{2}} f\right\|_{2} .
$$

Going back (4.38), we arrive at

$$
\begin{aligned}
& \frac{1}{\Delta t}\left(\mathcal{H}^{n+1}-\mathcal{H}^{n}\right)+\frac{5}{16}\left\|L_{N}\left(\frac{3}{4} e^{n+1}+\frac{1}{4} e^{n-1}\right)\right\|_{2}^{2} \\
\leq & \tilde{C}_{11}\left|\tilde{r}^{n+1}\right|^{2}+\tilde{C}_{12} \varepsilon^{-2}\left(\left\|L_{N}^{\frac{1}{2}} e^{n}\right\|_{2}^{2}+\left\|L_{N}^{\frac{1}{2}} e^{n-1}\right\|_{2}^{2}\right)+6\left\|\tau_{\phi}^{n+1}\right\|_{2}^{2}+\left|\tau_{r}^{n+1}\right|_{2}^{2} \\
\leq & \tilde{C}_{11}\left|\tilde{r}^{n+1}\right|^{2}+2 \tilde{C}_{12} \varepsilon^{-2}\left(\mathcal{H}^{n}+\mathcal{H}^{n-1}\right)+6\left\|\tau_{\phi}^{n+1 / 2}\right\|_{2}^{2}+\left|\tau_{r}^{n+1 / 2}\right|_{2}^{2}, \\
& \text { with } \mathcal{H}^{n+1}:=\frac{1}{2}\left\|L_{N}^{\frac{1}{2}} e^{n+1}\right\|_{2}^{2}+\frac{1}{8}\left\|L_{N}^{\frac{1}{2}}\left(e^{n+1}-e^{n}\right)\right\|_{2}^{2}+\left|\tilde{r}^{n+1}\right|^{2} .
\end{aligned}
$$

Therefore, with an application of discrete Gronwall inequality, and making use of the fact that

$$
\left\|\tau_{\phi}^{n+1 / 2}\right\|_{2,}, \tau_{r}^{n+1 / 2} \mid \leq C\left(\Delta t^{2}+h^{m}\right)
$$

we arrive at

$$
\mathcal{H}^{n+1}+\frac{5}{16} \Delta t \sum_{j=1}^{n}\left\|L_{N}\left(\frac{3}{4} e^{j+1}+\frac{1}{4} e^{j-1}\right)\right\|_{2}^{2} \leq \hat{C}\left(\Delta t^{4}+h^{2 m}\right),
$$

with $\hat{C}$ independent on $\Delta t$ and $h$. Meanwhile, the following summation estimate is available:

$$
\begin{aligned}
& \sum_{j=1}^{n}\left\|L_{N}\left(\frac{3}{4} e^{j+1}+\frac{1}{4} e^{j-1}\right)\right\|_{2}^{2} \geq \sum_{j=1}^{n}\left(\frac{3}{8}\left\|L_{N} \frac{3}{4} e^{j+1}\right\|_{2}^{2}-\frac{1}{8}\left\|L_{N} e^{j-1}\right\|_{2}^{2}\right) \\
\geq & \frac{1}{4} \sum_{j=0}^{n}\left\|L_{N} e^{j+1}\right\|_{2}^{2}-C\left(\Delta t^{4}+h^{2 m}\right) \geq \frac{\varepsilon^{4}}{4} \sum_{j=0}^{n}\left\|\Delta_{N}^{2} e^{j+1}\right\|_{2}^{2}-C\left(\Delta t^{4}+h^{2 m}\right) .
\end{aligned}
$$

In turn, the desired convergence estimate is available

$$
\left\|\Delta_{N} e^{n+1}\right\|_{2}+\left(\Delta t \sum_{j=1}^{k+1}\left\|\Delta_{N}^{2} e^{j}\right\|_{2}^{2}\right)^{\frac{1}{2}} \leq C \hat{C}^{\frac{1}{2}}\left(\Delta t^{2}+h^{m}\right),
$$

in which the equality,

$$
\left\|L_{N}^{\frac{1}{2}} e^{n+1}\right\|_{2}=\varepsilon\left\|\Delta_{N} e^{n+1}\right\|_{2}
$$

has been recalled. This completes the proof of Theorem 4.1. 
Remark 4.1. In an earlier error analysis work [45] for the SAV scheme applied to the Cahn-Hilliard flow, a linear refinement requirement for the time step size, $\Delta t \leq C h$, has to be imposed for the convergence estimate, since an inverse inequality has to be applied in the error estimate in the energy norm. In contrast, we have derived a higher order $H^{3}$ bound for the numerical solution, which in turn leads to an unconditional convergence estimate (no scaling law constraint between $\Delta t$ and $h$ ) for the SAV scheme applied to the SS equation.

Remark 4.2. As can be seen in the proof of Lemma 4.1, the uniform in time $H^{3}$ estimate (3.1) (for the numerical solution) plays an essential role in the derivation of the preliminary inequalities (4.9c), (4.9d). These two inequalities turn out to be very useful in the optimal rate convergence analysis for the proposed SAV scheme, since the error estimate has to be carried out in the $\ell^{\infty}\left(0, T ; H_{N}^{2}\right) \cap \ell^{2}\left(0, T ; H_{N}^{4}\right)$ norm. Instead, if the standard Crank-Nicolson approximation is applied to the surface diffusion term, such a uniform in time $H^{3}$ estimate is not theoretically available, as argued in Remark 3.2. As a result, the convergence analysis and error estimate for the standard Crank-Nicolson would not be theoretically established.

Remark 4.3. For the NSS equation (1.5) with the physical energy (1.4), the corresponding SAV scheme could be similar derived and analyzed. For example, the following inequality turns out to be valid, with an application of elliptic regularity:

$$
\int_{\Omega}\left(-\frac{1}{2} \ln \left(1+|\nabla \phi|^{2}\right)+\frac{\varepsilon^{2}}{4}|\Delta \phi|^{2}+C_{\varepsilon}\right) d \mathbf{x} \geq|\Omega|,
$$

in which $C_{\varepsilon}$ only depends on $\varepsilon$. In turn, one could denote

$$
E(\phi)=E_{1}(\phi)+\frac{1}{2}(\phi, L \phi)
$$

with

$$
E_{1}(\phi)=\int_{\Omega}\left(-\frac{1}{2} \ln \left(1+|\nabla \phi|^{2}\right)+\frac{\varepsilon^{2}}{4}|\Delta \phi|^{2}+C_{\varepsilon}\right) d \mathbf{x}, \quad L \phi=\frac{\varepsilon^{2}}{2} \Delta^{2} \phi .
$$

The corresponding SAV scheme could be designed by this energy decomposition, using similar ideas. The modified energy stability, uniform in time $H^{3}$ estimate for the numerical solution, and the optimal rate convergence analysis could be derived in a similar manner. This work will be left to the future works.

Remark 4.4. Other than the Crank-Nicolson method, some alternate second order temporal approximation, such as the second order BDF scheme, could be applied to the SAV scheme. In a recent work [60], a second order accurate SAV-BDF2 scheme has been successfully applied to the square phase field crystal (SPFC) model, in which the energy stability and optimal rate convergence analysis have been theoretically established. An application of the BDF2 temporal discretization to the SAV formulation (2.23) of the SS equation is expected to be feasible, with all the theoretical results available. The technical details are left to interested readers. 


\section{$5 \quad$ Numerical results}

In this section we present some numerical simulation results to demonstrate the stability and accuracy of the proposed scheme (2.24).

\subsection{Convergence tests}

We test the temporal convergence rate of the numerical scheme (2.24). The exact solution is taken as

$$
\phi(x, y, t)=\left(\frac{\sin (2 x) \cos (2 y)}{4}+0.48\right)\left(1-\frac{\sin ^{2}(t)}{2}\right)
$$

The computational domain is given by $(0,2 \pi)^{2}$, and interface width is set as $\varepsilon^{2}=0.1$. We use $128^{2}$ Fourier modes in space. From Fig. 1, the second order temporal convergence order has been clearly observed for the numerical scheme (2.24).

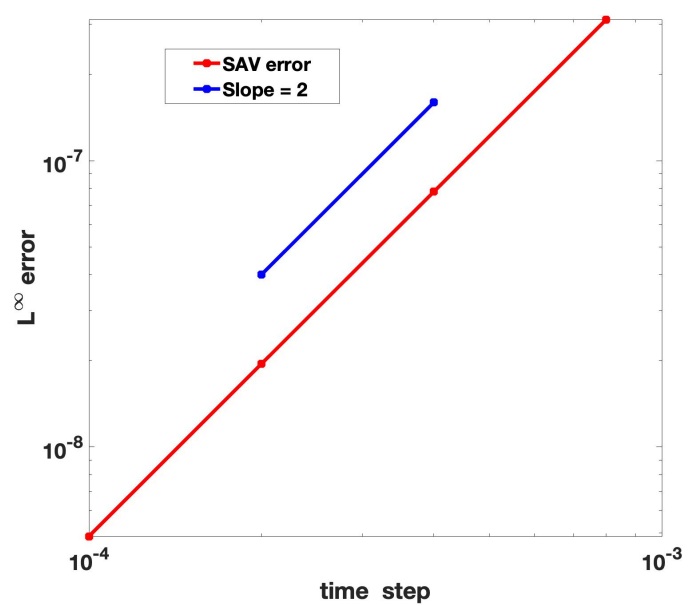

Figure 1: Convergence rate of SAV scheme (2.24) in time. The numerical error data is parallel to the curve of $\Delta t^{2}$.

\subsection{The coarsening dynamics}

In this subsection, we provide the coarsening process numerical simulation results for the MBE model (1.3) with slope selection. A random initial data is taken, varying from -0.001 to 0.001 . The physical parameters are set as

$$
\varepsilon=0.03, \quad M=1 .
$$



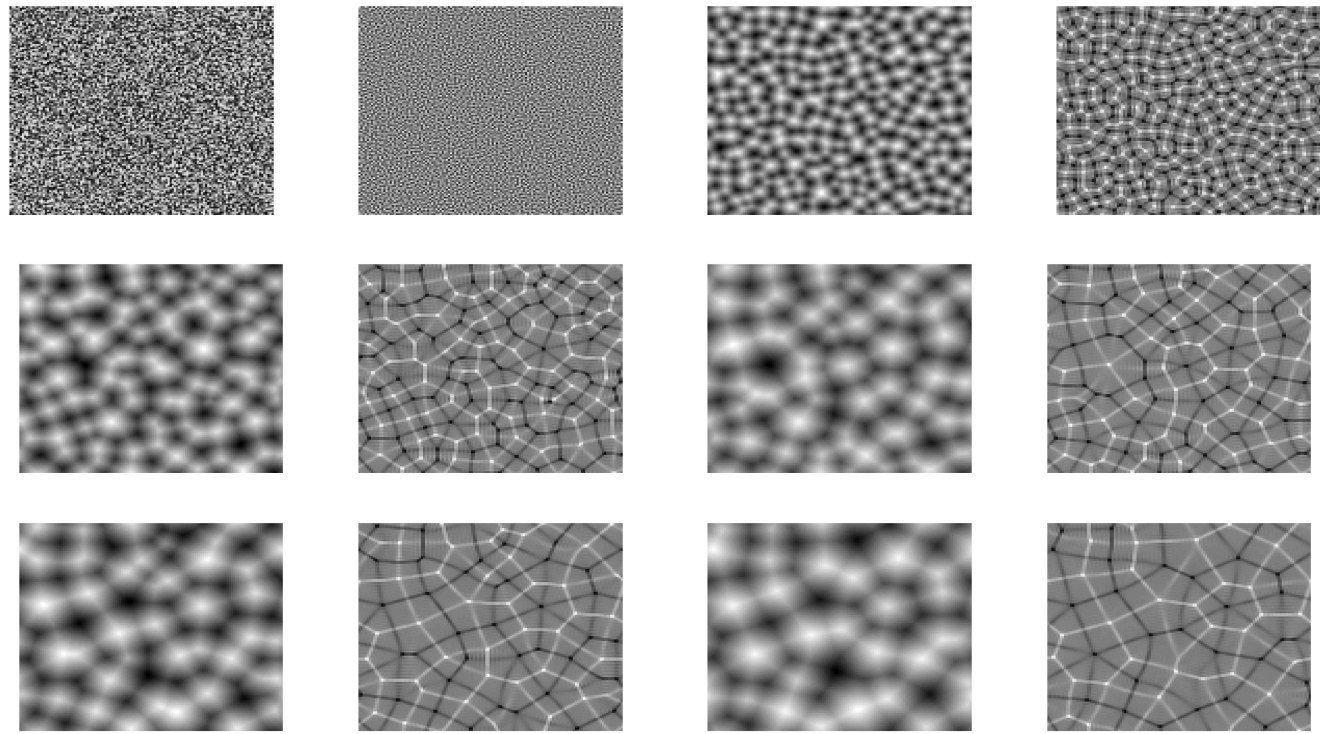

Figure 2: The isolines of the numerical solutions of the height function $\phi$ and its Laplacian $\Delta \phi$ for the model with slope selection with random initial data. For each subfigure, the left is $\phi$ and the right is $\Delta \phi$. Snapshots are taken at $t=0,2,20,40,60,100$, respectively.
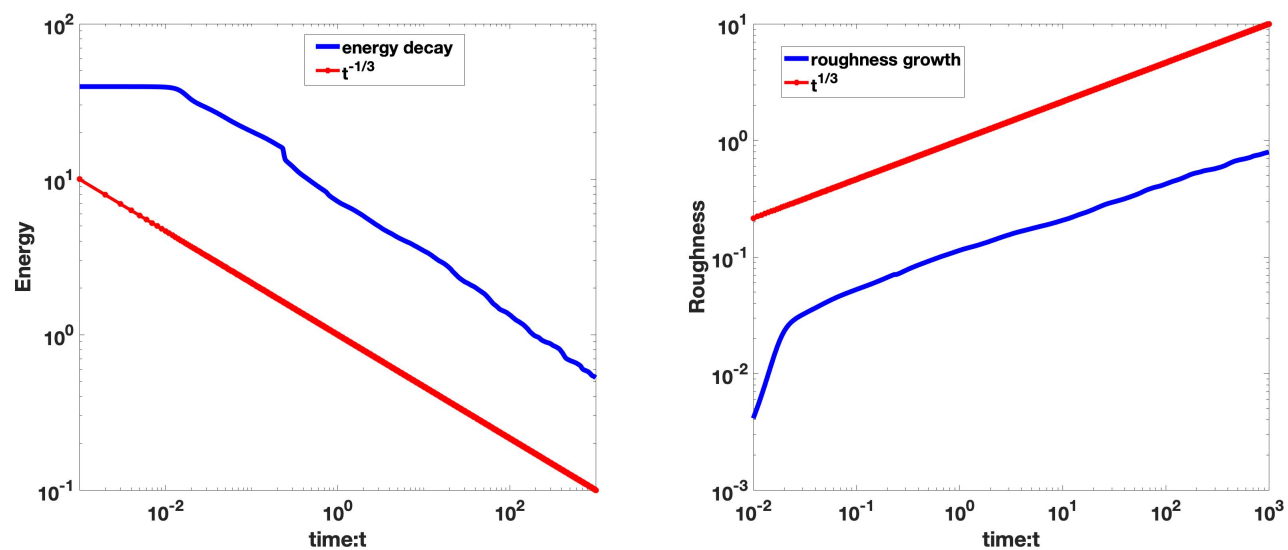

Figure 3: Dynamics of energy and roughness with respect to time. The energy evolution is parallel to the $t^{-\frac{1}{3}}$ curve, while the roughness evolution is parallel to the $t^{\frac{1}{3}}$ curve.

The computational domain is given by $\Omega=(0,12.8)^{2}$, and we use $128^{2}$ Fourier modes so that the numerical errors from the spatial discretization are negligibly small, in comparison with the temporal discretization error. The snapshot plots of the physical variable at a sequence of time instants, at $t=0,20,40,60,100$, computed by the numerical scheme (2.24), are displayed in Fig. 2. In addition, the time evolutions of the energy, as well as the 
roughness growth, are displayed in Fig. 3. A very nice agreement with the scaling laws, as given by $t^{-\frac{1}{3}}$ and $t^{\frac{1}{3}}$, respectively [37], is observed.

\section{Concluding remarks}

In this article, we have analyzed a scalar auxiliary variable (SAV)-based numerical scheme for slope-selection (SS) equation of the epitaxial thin film growth model, with Fourier pseudo-spectral spatial approximation. In particular, the standard Crank-Nicolson approximation to the surface diffusion term is replaced by a modified version, with $3 / 4$ and $1 / 4$ coefficient distribution at time steps $t^{n+1}, t^{n-1}$, respectively. With an application of this approximation to the second order SAV scheme for the SS equation, a modified energy stability is proved, so that a uniform $\mathrm{H}^{2}$ bound for the numerical solution (of the phase variable) is available. In addition, a higher order $H^{3}$ estimate has also been derived, with the help of various discrete Sobolev inequality in the Fourier pseudo-spectral space. With such an $H^{3}$ bound at hand, we have derived an optimal rate $\left(\mathcal{O}\left(\Delta t^{2}+h^{m}\right)\right)$ convergence analysis for the numerical scheme in the energy norm, i.e., in the $\ell^{\infty}\left(0, T ; H_{N}^{2}\right) \cap \ell^{2}\left(0, T ; H_{N}^{4}\right)$ norm. In particular, the aliasing error control techniques have to be applied in the nonlinear error estimate associated with the 4-Laplacian term. A few numerical experiments are also presented, which confirm the efficiency and accuracy of the proposed scheme.

\section{Acknowledgements}

This work is supported in part by NSF DMS-2012669 (C. Wang).

\section{References}

[1] R. Backofen, S. M. Wise, M. Salvalaglio, And A. Voigt, Convexity splitting in a phase field model for surface diffusion, Int. J. Numer. Anal. Model., 16 (2019), pp. 192-209.

[2] J. P. BOYD, Chebyshev and Fourier Spectral Methods, Courier Corporation, 2001.

[3] C. CANUTO AND A. QUARTERONI, Approximation results for orthogonal polynomials in Sobolev spaces, Math. Comput., 38 (1982), pp. 67-86.

[4] N. CHEN, C. WANG, AND S. M. WISE, Global-in-time Gevrey regularity solution for a class of bistable gradient flows, Discrete Contin. Dyn. Syst. Ser. B, 21 (2016), pp. 1689-1711.

[5] W. Chen, S. COnde, C. WANG, X. WANG, AND S. M. WISE, A linear energy stable scheme for a thin film model without slope selection, J. Sci. Comput., 52 (2012), pp. 546-562.

[6] W. CHEN, W. FEnG, Y. LiU, C. WANG, AND S. M. WISE, A second order energy stable scheme for the Cahn-Hilliard-Hele-Shaw equation, Discrete Contin. Dyn. Syst. Ser. B, 24(1) (2019), pp. 149-182.

[7] W. CHen, W. LI, Z. LUO, C. WANG, AND X. WANG, A stabilized second order exponential time differencing multistep method for thin film growth model without slope selection, EASIM Math. Model. Numer. Anal., 54 (2020), pp. 727-750. 
[8] W. CHEN, W. LI, C. WANG, S. WANG, AND X. WANG, Energy stable higher order linear ETD multi-step methods for gradient flows: application to thin film epitaxy, Res. Math. Sci., 7 (2020), 13.

[9] W. CHEN, Y. LIU, C. WANG, AND S. M. WISE, An optimal-rate convergence analysis of a fully discrete finite difference scheme for Cahn-Hilliard-Hele-Shaw equation, Math. Comput., 85 (2016), pp. 2231-2257.

[10] W. CHEN, C. WANG, X. WANG, AND S. M. WISE, A linear iteration algorithm for energy stable second order scheme for a thin film model without slope selection, J. Sci. Comput., 59 (2014), pp. 574-601.

[11] W. CHEN AND Y. WANG, A mixed finite element method for thin film epitaxy, Numer. Math., 122 (2012), pp. 771-793.

[12] K. Cheng, W. Feng, S. GotTlieb, And C. WANG, A Fourier pseudospectral method for the "Good" Boussinesq equation with second-order temporal accuracy, Numer. Methods Partial Differential Equations, 31(1) (2015), pp. 202-224.

[13] K. Cheng, Z. QIAO, AND C. WANG, A third order exponential time differencing numerical scheme for no-slope-selection epitaxial thin film model with energy stability, J. Sci. Comput., 81(1) (2019), pp. 154-185.

[14] K. CHENG AND C. WANG, Long time stability of high order multi-step numerical schemes for two-dimensional incompressible Navier-Stokes equations, SIAM J. Numer. Anal., 54 (2016), pp. 3123-3144.

[15] K. Cheng, C. WANG, AND S. M. WISE, An energy stable fourier pseudo-spectral numerical scheme for the square phase field crystal equation, Commun. Comput. Phys., 26 (2019), pp. 13351364.

[16] K. Cheng, C. WANG, S. M. WISE, AND X. YUE, A second-order, weakly energy-stable pseudospectral scheme for the Cahn-Hilliard equation and its solution by the homogeneous linear iteration method, J. Sci. Comput., 69 (2016), pp. 1083-1114.

[17] Q. CHENG, J. SHEN, AND X. YANG, Highly efficient and accurate numerical schemes for the epitaxial thin film growth models by using the SAV approach, J. Sci. Comput., 78 (2018), pp. 14671487.

[18] A. DIEGEL, X. FENG, AND S. M. WISE, Convergence analysis of an unconditionally stable method for a Cahn-Hilliard-Stokes system of equations, SIAM J. Numer. Anal., 53 (2015), pp. 127-152.

[19] A. DiEGEL, C. WANG, X. WANG, AND S. M. WISE, Convergence analysis and error estimates for a second order accurate finite element method for the Cahn-Hilliard-Navier-Stokes system, Numer. Math., 137 (2017), pp. 495-534.

[20] A. DIEGEL, C. WANG, AND S. M. WISE, Stability and convergence of a second order mixed finite element method for the Cahn-Hilliard equation, IMA J. Numer. Anal., 36 (2016), pp. 1867-1897.

[21] W. E, Convergence of spectral methods for the Burgers equation, SIAM J. Numer. Anal., 29 (1992), pp. 1520-1541.

[22] W. E, Convergence of Fourier methods for Navier-Stokes equations, SIAM J. Numer. Anal., 30 (1993), pp. 650-674.

[23] G. EHRLICH AND F. G. HudDA, Atomic view of surface diffusion: Tungsten on tungsten, J. Chem. Phys., 44 (1966), pp. 1036-1099.

[24] W. Feng, A. J. SAlgAdo, C. WANG, AND S. M. Wise, Preconditioned steepest descent methods for some nonlinear elliptic equations involving p-Laplacian terms, J. Comput. Phys., 334 (2017), pp. 45-67.

[25] W. FENG, C. WANG, S. M. WISE, AND Z. ZHANG, A second-order energy stable Backward Differentiation Formula method for the epitaxial thin film equation with slope selection, Numer. Methods Partial Differential Equations, 34(6) (2018), pp. 1975-2007. 
[26] Y. GONG, J. ZHAO, AND Q. WANG, An efficient and unconditionally energy stable scheme for simulating solid-state dewetting of thin films with isotropic surface energy, Commun. Comput. Phys., 26 (2019), pp. 1444-1470.

[27] D. Gottlieb And S. A. Orszag, Numerical Analysis of Spectral Methods, Theory and Applications, SIAM, Philadelphia, PA, 1977.

[28] S. Gottlieb, F. TONe, C. WANG, X. WANG, AND D. Wirosoetisno, Long time stability of a classical efficient scheme for two dimensional Navier-Stokes equations, SIAM J. Numer. Anal., 50 (2012), pp. 126-150.

[29] S. GotTlieB AND C. WANG, Stability and convergence analysis of fully discrete Fourier collocation spectral method for 3-d viscous Burgers' equation, J. Sci. Comput., 53 (2012), pp. 102-128.

[30] Z. GUAN, J. S. LOWENGRUB, AND C. WANG, Convergence analysis for second order accurate schemes for the periodic nonlocal Allen-Cahn and Cahn-Hilliard equations, Math. Methods Appl. Sci., 40(18) (2017), pp. 6836-6863.

[31] Z. Guan, J. S. Lowengrub, C. WAnG, And S. M. WiSe, Second-order convex splitting schemes for nonlocal Cahn-Hilliard and Allen-Cahn equations, J. Comput. Phys., 277 (2014), pp. $48-71$.

[32] J. GUO, C. WANG, S. M. WISE, AND X. YUE, An $H^{2}$ convergence of a second-order convexsplitting, finite difference scheme for the three-dimensional Cahn-Hilliard equation, Commun. Math. Sci., 14 (2016), pp. 489-515.

[33] Y. HAO, Q. HUANG, AND C. WANG, A third order BDF energy stable linear scheme for the no-slope-selection thin film model, Commun. Comput. Phys., 29 (2021), pp. 905-929.

[34] J. S. Hesthaven, S. Gottlieb, And D. Gottlieb, Spectral Methods for Time-Dependent Problems, volume 21, Cambridge University Press, 2007.

[35] L. JU, X. LI, Z. QIAO, AND H. ZHANG, Energy stability and convergence of exponential time differencing schemes for the epitaxial growth model without slope selection, Math. Comput., 87 (2018), pp. 1859-1885.

[36] R. V. KOHN, Energy-driven pattern formation, Proceedings of the International Congress of Mathematicians, M. Sanz-Sole, J. Soria, J. L. Varona, and J. Verdera, eds., European Mathematical Society Publishing House, Madrid, 1 (2007), pp. 359-384.

[37] R. V. KOHN AND X. YAN, Upper bound on the coarsening rate for an epitaxial growth model, Commun. Pure Appl. Math., 56 (2003), pp. 1549-1564.

[38] B. LI, High-order surface relaxation versus the Ehrlich-Schwoebel effect, Nonlinearity, 19 (2006), pp. 2581-2603.

[39] B. Li AND J.-G. LIU, Thin film epitaxy with or without slope selection, Euro. J. Appl. Math., 14 (2003), pp. 713-743.

[40] B. LI AND J.-G. LIU, Epitaxial growth without slope selection: energetics, coarsening, and dynamic scaling, J. Nonlinear Sci., 14 (2004), pp. 429-451.

[41] D. Li AND Z. QIAO, On second order semi-implicit Fourier spectral methods for 2D Cahn-Hilliard equations, J. Sci. Comput., 70 (2017), pp. 301-341.

[42] D. LI AND Z. QIAO, On the stabilization size of semi-implicit Fourier-spectral methods for 3D Cahn-Hilliard equations, Commun. Math. Sci., 15 (2017), pp. 1489-1506.

[43] D. LI, Z. QIAO, AND T. TANG, Characterizing the stabilization size for semi-implicit Fourierspectral method to phase field equations, SIAM J. Numer. Anal., 54 (2016), pp. 1653-1681.

[44] W. LI, W. CHEN, C. WANG, Y. YAN, AND R. HE, A second order energy stable linear scheme for a thin film model without slope selection, J. Sci. Comput., 76(3) (2018), pp. 1905-1937.

[45] X. LI, J. SHEN, AND H. RUI, Energy stability and convergence of SAV block-centered finite difference method for gradient flows, Math. Comput., 88 (2019), pp. 2047-2068. 
[46] H. LiaO, X. SONG, T. TANG, AND T. ZHOU, Analysis of the second order BDF scheme with variable steps for the molecular beam epitaxial model without slope selection, Sci. China Math., (2021), accepted and published online: https://link. springer.com/article/10.1007/s11425-020-1817-4.

[47] Y. LIU, W. CHEN, C. WANG, AND S. M. WISE, Error analysis of a mixed finite element method for a Cahn-Hilliard-Hele-Shaw system, Numer. Math., 135 (2017), pp. 679-709.

[48] X. MENG, Z. QIAO, C. WANG, AND Z. ZHANG, Artificial regularization parameter analysis for the no-slope-selection epitaxial thin film model, CSIAM Trans. Appl. Math., 1 (2020), pp. 441-462.

[49] D. MOldovan AND L. GOlUBOVIC, Interfacial coarsening dynamics in epitaxial growth with slope selection, Phys. Rev. E, 61 (2000), 6190.

[50] Z. QIAO, Z. SUN, AND Z. ZHANG, The stability and convergence of two linearized finite difference schemes for the nonlinear epitaxial growth model, Numer. Methods Partial Differential Equations, 28 (2012), pp. 1893-1915.

[51] Z. QIAO, Z. SUN, AND Z. ZHANG, Stability and convergence of second-order schemes for the nonlinear epitaxial growth model without slope selection, Math. Comput., 84 (2015), pp. 653-674.

[52] Z. QIAO, C. WANG, S. M. WISE, AND Z. ZHANG, Error analysis of a finite difference scheme for the epitaxial thin film growth model with slope selection with an improved convergence constant, Int. J. Numer. Anal. Model., 14 (2017), pp. 283-305.

[53] Z. QIAO, Z. ZHANG, AND T. TANG, An adaptive time-stepping strategy for the molecular beam epitaxy models, SIAM J. Sci. Comput., 33 (2012), pp. 1395-1414.

[54] R. L. SchWOeBel, Step motion on crystal surfaces: II, J. Appl. Phys., 40 (1969), pp. 614-618.

[55] J. SHEN, C. WANG, X. WANG, AND S. M. WISE, Second-order convex splitting schemes for gradient flows with Ehrlich-Schwoebel type energy: Application to thin film epitaxy, SIAM J. Numer. Anal., 50 (2012), pp. 105-125.

[56] J. SHEN AND J. XU, Convergence and error analysis for the scalar auxiliary variable (SAV) schemes to gradient flows, SIAM J. Numer. Anal., 56 (2018), pp. 2895-2912.

[57] J. SHEN, J. XU, AND J. YANG, The scalar auxiliary variable (SAV) approach for gradient flows, J. Comput. Phys., 353 (2018), pp. 407-416.

[58] J. SHEN, J. XU, AND J. YANG, A new class of efficient and robust energy stable schemes for gradient flows, SIAM Review, 61(3) (2019), pp. 474-506.

[59] C. WANG, X. WANG, AND S. M. WISE, Unconditionally stable schemes for equations of thin film epitaxy, Discrete Contin. Dyn. Sys. A, 28 (2010), pp. 405-423.

[60] M. WANG, Q. HUANG, AND C. WANG, A second order accurate scalar auxiliary variable (SAV) numerical method for the square phase field crystal equation, J. Sci. Comput., (2021), accepted and in press, https://arxiv.org/abs/2012.15133.

[61] S. WANG, W. CHEN, H. PAN, AND C. WANG, Optimal rate convergence analysis of a second order scheme for a thin film model with slope selection, J. Comput. Appl. Math., 377 (2020), 112855.

[62] X. WANG, An efficient second order in time scheme for approximating long time statistical properties of the two dimensional Navier-Stokes equations global smooth solutions of the modified phase field crystal equation, Methods Appl. Anal., 17 (2010), pp. 191-212.

[63] C. XU AND T. TANG, Stability analysis of large time-stepping methods for epitaxial growth models, SIAM J. Numer. Anal., 44 (2006), pp. 1759-1779.

[64] X. YANG, J. ZHAO, AND Q. WANG, Numerical approximations for the molecular beam epitaxial growth model based on the invariant energy quadratization method, J. Comput. Phys., 333 (2017), pp. 104-127.

[65] C. ZHANG, J. HUANG, C. WANG, AND X. YUE, On the operator splitting and integral equation preconditioned deferred correction methods for the "Good" Boussinesq equation, J. Sci. Comput., 75 
(2018), pp. 687-712.

[66] C. ZhANG, H. WANG, J. HuAnG, C. WANG, AND X. YUE, A second order operator splitting numerical scheme for the "Good" Boussinesq equation, Appl. Numer. Math., 119 (2017), pp. 179193.

[67] C. H. ZhANG, J. OUYANG, C. WANG, AND S. M. Wise, Numerical comparison of modifiedenergy stable SAV-type schemes and classical BDF methods on benchmark problems for the functionalized Cahn-Hilliard equation, J. Comput. Phys., 423 (2020), 109772. 